\title{
NADPH Oxidases Are Involved in Differentiation and Pathogenicity in Botrytis cinerea
}

\author{
Nadja Segmüller, ${ }^{1}$ Leonie Kokkelink, ${ }^{1}$ Sabine Giesbert, ${ }^{1}$ Daniela Odinius, ${ }^{1}$ Jan van Kan, ${ }^{2}$ and \\ Paul Tudzynski ${ }^{1}$
}

${ }^{1}$ Institut für Botanik, Westf. Wilhelms-Universität, Schlossgarten 3, D-48149 Münster, Germany; ${ }^{2}$ Laboratory of Phytopathology, Wageningen University, P.O. Box 80 25, NL-6700 EE Wageningen, The Netherlands

Submitted 5 November 2007. Accepted 13 February 2008.

\begin{abstract}
Nicotinamide adenine dinucleotide (NADPH) oxidases have been shown to be involved in various differentiation processes in fungi. We investigated the role of two NADPH oxidases in the necrotrophic phytopathogenic fungus, Botrytis cinerea. The genes bcnox $A$ and bcnoxB were cloned and characterized; their deduced amino acid sequences show high homology to fungal NADPH oxidases. Analyses of single and double knock-out mutants of both NADPH oxidase genes showed that both bcnoxA and bcnoxB are involved in formation of sclerotia. Both genes have a great impact on pathogenicity: whereas bcnoxB mutants showed a retarded formation of primary lesions, probably due to an impaired formation of penetration structures, bcnox $A$ mutants were able to penetrate host tissue in the same way as the wild type but were much slower in colonizing the host tissue. Double mutants showed an additive effect: they were aberrant in penetration and colonization of plant tissue and, therefore, almost nonpathogenic. To study the structure of the fungal Nox complex in more detail, bcnox $R$ (encoding a homolog of the mammalian $p 67^{\text {phox }}$, a regulatory subunit of the Nox complex) was functionally characterized. The phenotype of $\triangle$ bcnoxR mutants is identical to that of $\triangle$ bcnoxAB double mutants, providing evidence that Bcnox $R$ is involved in activation of both Bcnox enzymes.
\end{abstract}

Additional keywords: reactive oxygen species (ROS).

Generation of reactive oxygen species (ROS) is a characteristic feature of defense reactions of eukaryotic cells against pathogen attack (Baker and Orlandi 1995; Lamb and Dixon 1997; Segal 2005; Wientjes and Segal 1995; Wojtaszek 1997). In neutrophils and macrophages of mammals, as well as in plant cells, hydrogen peroxide and other reactive oxidants are used in defense reactions against bacteria and fungi (Bedard and Krause 2007; Torres et al. 2006). The key enzyme in defense reactions against pathogens that triggers the oxidative burst seems to be the nicotinamide adenine dinucleotide (NADPH) oxidase. This multisubunit complex generates superoxide $\left(\mathrm{O}_{2}^{-}\right)$in the one-electron reduction of $\mathrm{O}_{2}$ using electrons supplied by NADPH (Cross and Jones 1991; Parkos et al.

Corresponding author: P. Tudzynski; Telephone.: +49-251-83 2 4998; Fax: +49-251-83 2 1601; E-mail: tudzyns@uni-muenster.de

Nucleotide sequence data is available under accession numbers: bcnoxA, AM900412; bcnoxB, AM900413; and bcnoxR, AM 899997.

* The $e$-Xtra logo stands for "electronic extra" and indicates three supplemental figures are published online.
1988). The phagocyte NADPH oxidase is composed of a catalytic subunit gp91 $1^{\text {phox }}$ (NOX2) and several regulatory subunits (e.g., p67 $7^{\text {phox }}$ and the GTPase Rac). The assembly of these regulatory subunits regulates the enzyme activity of $\mathrm{gp} 91^{\text {phox }}$. Heritable defects in certain regulatory subunits are the basis of chronic granulomatous disease, a disorder of white cell function characterized by recurrent, severe bacterial and fungal infections (Babior 1992; Dinauer 1993). In addition to the function of ROS in defense, these molecules are involved in regulating immunity, cell proliferation, cell differentiation, signal transduction, and ion transport (Finkel 2003; Foreman et al. 2003; Kwak et al. 2003; Lambeth 2004).

Recently, it also has been shown that microbial eukaryotes like fungi and algae possess NADPH oxidases homologous to gp91 ${ }^{\text {phox }}$ (Aguirre et al. 2005; Herve et al. 2006). It has been postulated that, in fungi, the existence of NADPH oxidases is linked to a multicellular life style. While unicellular yeasts do not possess nox genes, the number of nox genes increases from one in Aspergillus nidulans to two in Podospora anserina and to at least three in Magnaporthe grisea (Aguirre et al. 2005; Takemoto et al. 2007). Functional analyses of NADPH oxidases of A. nidulans (Lara-Ortiz et al. 2003) and P. anserina (Malagnac et al. 2004) showed that these enzymes are involved in sexual differentiation. The expression of noxA in A. nidulans is controlled by the stress-activated mitogen-activated protein kinase (MAPK) SakA, connecting stress-induced MAPK signaling to the regulation of ROS production. In the endophyte Epichloe festucae, inactivation of the noxA gene led to a switch from a mutualistic to antagonistic interaction with its host (Tanaka et al. 2006). Plants infected with the E. festucae noxA mutant showed disease symptoms and the fungal biomass increased dramatically. ROS accumulation was observed in the extracellular matrix of the endophyte and at the interface between extracellular matrix and host cell walls of meristematic tissue in the wild type but not in noxA mutants. Deletion of the second NADPH oxidase gene in this fungus, noxB, had no effect (Tanaka et al. 2006). Similarly, in the phytopathogenic fungus Claviceps purpurea, which is considered a biotroph, Cpnox1 is required for normal pathogenic development, whereas deletion of Cpnox 2 had no influence on pathogenicity (Giesbert et al. in press; S. Giesbert, D. Buttermann, T. Schuerg, S. Scheele, and P. Tudzynski, unpublished data). In contrast, in the phytopathogenic fungus $M$. grisea, Nox1 and Nox 2 both are required independently for pathogenicity (Egan et al. 2007). Mutants of both NADPH oxidase genes in this fungus are impaired in appressorium-mediated cuticle penetration, and therefore, incapable of causing plant disease. Analyses of the ROS status during differentiation of appressoria revealed that there was no detectable reduction of superoxide 
production in either noxl or nox 2 mutants, whereas the double knock-out mutant showed less $\mathrm{O}_{2}^{-}$in the appressorium compared with the wild-type strain. Interestingly, the amount of $\mathrm{O}_{2}^{-}$increased in growing hyphal tips of the double mutant compared with the wild type, indicating that there is an alternative ROS source that is activated upon loss of the Nox enzymes. In $P$. anserina, enhanced ROS production in mycelia also was found in PaNox deletion strains (Malagnac et al. 2004).

The plant pathogen Botrytis cinerea causes gray mold disease in a broad range of crop plants. Because of its necrotrophic lifestyle, the fungus depends essentially on its ability to kill host cells before colonizing the plant tissue (Williamson et al. 2007). Von Tiedemann (1997) showed that B. cinerea induces a significant oxidative burst and that the virulence of the fungal isolates correlates positively with the intensity of this oxidative burst. There is evidence that $B$. cinerea may even require the hypersensitive response (HR) of its host plant to achieve full pathogenicity (Govrin and Levine 2000). Therefore, $B$. cinerea produces ROS in axenic culture and in planta: cytochemical analysis showed the presence of $\mathrm{O}_{2}^{-}$in hyphal tips and $\mathrm{H}_{2} \mathrm{O}_{2}$ generation in and around the penetrated host cell wall (Tenberge et al. 2002). There is further clear evidence for ROS and the occurrence of oxidative stress in tissues attacked by $B$. cinerea (Lyon et al. 2004). Therefore, the presence of NADPH oxidases in filamentous fungi is of great interest. They are considered to be key enzymes in the production of ROS and, therefore, could play an important part in virulence of $B$. cinerea by generating $\mathrm{O}_{2}^{-}$, which can be converted further by superoxide dismutases (SOD) to $\mathrm{H}_{2} \mathrm{O}_{2}$. A superoxide dismutase of $B$. cinerea (Bcsod1) was analyzed by Rolke and associates (2004) and shown to be a virulence factor, indicating that the $\mathrm{H}_{2} \mathrm{O}_{2}$ production via $\mathrm{Cu}-\mathrm{Zn}-\mathrm{SOD}$ from an internal (?) $\mathrm{O}_{2}^{-}$source has an effect on virulence. A possible source for this $\mathrm{O}_{2}^{-}$could be an NADPH oxidase.

Here, we present the isolation of $B$. cinerea bcnoxA and bcnox $B$ genes, which encode two NADPH oxidases that show homology to NoxA (bcnoxA) and to NoxB (bcnoxB) proteins described by Aguirre and associates (2005). We show that both NADPH oxidases have significant impact on differentiation and pathogenesis. In addition, functional analysis of the bcnoxR gene encoding a homolog of a regulatory subunit of the mammalian Nox complex shows that BcnoxR is required for activation of both catalytic subunits in $B$. cinerea.

\section{RESULTS}

bcnoxA and bcnoxB encode two NADPH oxidases.

An expressed sequence tag library from an axenic culture of B. cinerea strain American Type Culture Collection 58025 (Siewers et al. 2005) contained a cDNA clone with significant homology to NADPH oxidase PaNox2 of $P$. anserina, which was used to probe a genomic EMBL3 library of $B$. cinerea SAS56. Hybridizing lambda clones were subcloned and sequenced. A genomic 10-kb SalI fragment contained the coding region of an NADPH oxidase gene (termed bcnoxB). The bcnox $B$ sequence of $1,860 \mathrm{bp}$ contains an open reading frame (ORF) of 1,748 bp interrupted by two introns. The deduced bcnoxB protein product (BcnoxB) consists of 581 amino acids, with an estimated molecular mass of $66.15 \mathrm{kDa}$ and an isoelectric point $(\mathrm{pI})$ of 9.11 . BcnoxB has significant similarity to PaNox 2 of $P$. anserina, Nox 2 of $C$. purpurea, NoxB of E. festucae, and Nox 2 of M. grisea (Fig. 1).

Using the sequences of PaNoxl and noxA of A. nidulans, the corresponding gene bcnoxA could be identified within the $B$. cinerea genomic database (BC1G_10823, Supercontig 80: 55019-56928). DNA sequence analysis revealed an ORF of $1,659 \mathrm{bp}$ interrupted by four introns. The presence of the introns was confirmed by reverse-transcriptase polymerase chain reaction (RT-PCR) analysis (data not shown). The deduced bcnoxA protein product (BcnoxA) consists of 553 amino acids, with an estimated molecular mass of $63.4 \mathrm{kDa}$ and a $\mathrm{pI}$ of 7.92. The BcnoxA sequence has significant similarity to NADPH oxidases of filamentous fungi, such as Cpnox 1 from C. purpurea, Panox 1 from $P$. anserina, NoxA from E. festucae, and Nox1 from M. grisea. (Fig. 1).

Both $B$. cinerea NADPH oxidases showed all the characteristic conserved domains such as NADPH- and FAD-binding domains and six transmembrane domains (database entries: AM 900412/AM 900413).

\section{bcnox $A$ is upregulated during oxidative stress.}

Transcriptional regulation of bcnox genes was investigated using mycelia grown under different stress conditions $(\mathrm{pH}$, oxidative, osmotic, and heat stress). Under standard growth conditions (Czapek Dox [CD] medium), the expression level of both genes was very low and remained unaltered during growth at different $\mathrm{pH}$ values (Fig. 2). Oxidative stress medi-
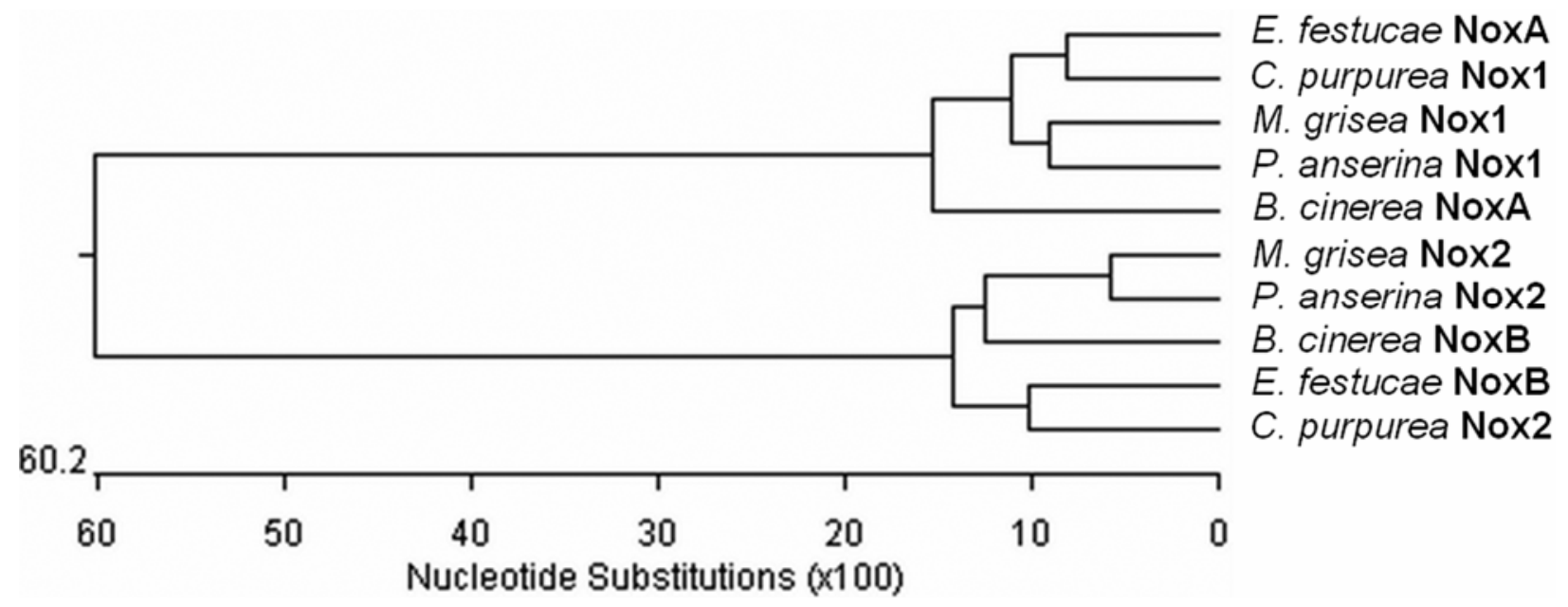

Fig. 1. Phylogenetic tree of nicotinamide adenine dinucleotide (NADPH)-oxidase sequences of fungi. Sequence alignment and tree construction were performed with ClustalW by use of MegAlign, a program of the Lasergene package (DNASTAR). GenBank accession numbers: Epichloe festucae NoxA (BAE72680), E. festucae NoxB (BAE72682), Claviceps purpurea Cpnox1 (CAP12327), C. purpurea Cpnox2 (CAP12328), Podospora anserina Nox1 (AAK50853), P. anserina Nox2 (AAQ74977), Botrytis cinerea BcnoxA (CAP12516), B. cinerea BcnoxB (CAP12517), Magnaporthe grisea Nox1 (EAA49092), and $M$. grisea Nox2 (EAA56588). 
ated by $5 \mathrm{mM}$ and $10 \mathrm{mM} \mathrm{H} \mathrm{H}_{2}$, as well as $500 \mu \mathrm{M}$ meadione, led to increased transcript levels of bcnoxA, whereas bcnoxB transcripts were barely affected by oxidative stress. The slight upregulation of bcnoxB during menadione and $\mathrm{NaCl}$ treatment in the experiment documented in Figure 2 was not significant; in most analyses (also in other contexts), the transcript levels of this gene were below detection level (here, three biological repeats). Osmotic stress $(0.8 \mathrm{M} \mathrm{NaCl})$ and temperature stress (growth at $28^{\circ} \mathrm{C}$ ) had no significant effect on transcript levels of bcnoxA or bcnoxB. Altogether, it appears that bcnoxA is upregulated during oxidative stress, whereas $\mathrm{pH}$, osmotic stress, and elevated temperature have no impact on the expression level. This is a rather unexpected result, because BcnoxA itself probably contributes to the ROS level. Conditions regulating the expression of bcnoxB could not be detected due to the very low expression level (Fig. 2).

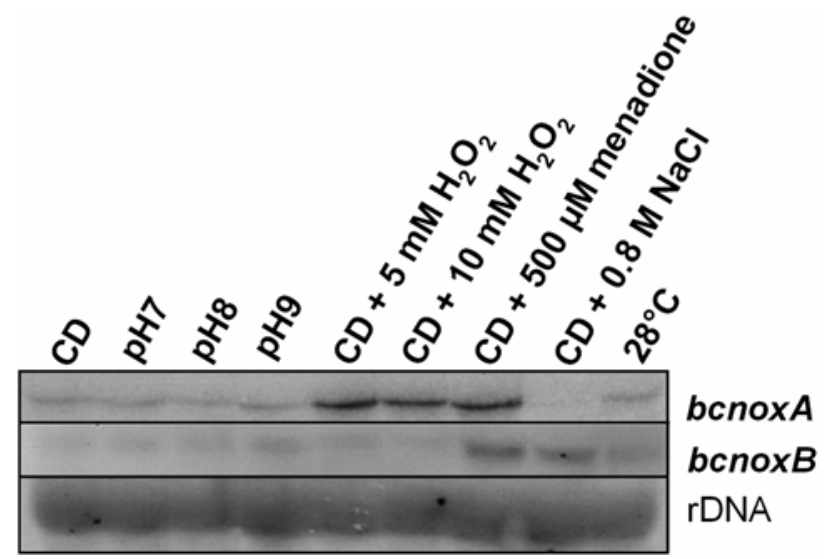

Fig. 2. Expression of bcnoxA and bcnoxB in axenic culture. Wild-type B05.10 was incubated in $1.5 \%$ malt medium for $24 \mathrm{~h}$ and transferred to Czapek Dox (CD) medium, pH 5.2, for $24 \mathrm{~h}$. Subsequently mycelia were shifted in $\mathrm{CD}$ medium with different $\mathrm{pH}$ or containing $\mathrm{H}_{2} \mathrm{O}_{2}$, menadione, or $\mathrm{NaCl}$ as indicated for $1 \mathrm{~h}$ at $18^{\circ} \mathrm{C}$. One culture was incubated for $1 \mathrm{~h}$ at $28^{\circ} \mathrm{C}$ (CD medium, $\mathrm{pH}$ 5.2). RNA was isolated and used for a Northern hybridization with bcnoxA or bcnoxB probes, respectively. As a loading control, the blot was hybridized with a Botrytis cinerea rDNA probe.

\section{Expression of both NADPH oxidase genes is controlled} by MAPK cascades.

In A. nidulans, the expression of noxA is controlled by the MAPK SakA (Lara-Ortiz et al. 2003); therefore, we studied the impact of MAPK cascades on expression of the bcnox genes in B. cinerea (Fig. 3). Expression levels of both bcnox genes were determined in mycelia grown under oxidative and osmotic stress conditions of the wild type and three MAPK mutants: $\Delta$ bmp1 (a homolog of $M$. grisea pmk1) (Doehlemann et al. 2006; Zheng et al. 2000), $\Delta$ bmp3 (corresponding to mps1) (Rui and Hahn 2007), and $\Delta$ bcsak1 (homolog of osm1) (Segmüller et al. 2007). In contrast to A. nidulans, Bcsak1 had no effect on expression of both bcnox genes. Bmp1 mutants displayed a stronger bcnoxA induction under oxidative stress. However, the most prominent effects were observed with the $\Delta$ bmp3 mutant: noxA induction by oxidative stress was virtually abolished, whereas noxB was highly expressed under all conditions (Fig. 3). These data suggest that Bmp1 had a slightly negative modulating effect on bcnoxA expression, whereas Bmp3 controlled bcnoxA expression positively and bcnoxB expression negatively. Thus, Bmp3 seems to have a key function in transcriptional regulation of the bcnox genes.

\section{Generation of $\Delta \mathrm{bcnox} A, \Delta \mathrm{bcnox} B$, and double mutants.}

For a functional analysis, bcnoxA and bcnoxB deletion mutants were created using a gene replacement approach. To yield the replacement vector $\mathrm{p} \triangle \mathrm{NOXA}$, the $5^{\prime}$ and $3^{\prime}$ flanking regions of the gene (433 and $554 \mathrm{bp}$, respectively) were amplified and cloned into the nourseothricin-resistance vector $\mathrm{pNR} 1$ (Fig. 4A; discussed in detail below). The replacement construct was excised with SacI and SalI and used to transform strain B05.10. Nourseothricin-resistant transformants were tested by PCR for homologous integration of the replacement fragment. The primer pair 1/2 (binding within the resistance cassette and outside the replacement fragment, respectively) (Fig. 4A) yielded a fragment of $1.275 \mathrm{~kb}$ only if the fragment had been integrated by double crossover at the homologous site. Of the 20 transformants obtained, 5 showed this diagnostic band. All these primary transformants were heterokaryons (as is normal in $B$. cinerea), because PCR with primer pair 3/4 yielded the fragment specific to the wild type $(1,031 \mathrm{bp})$. Five of the primary transformants were subjected to four rounds of single-

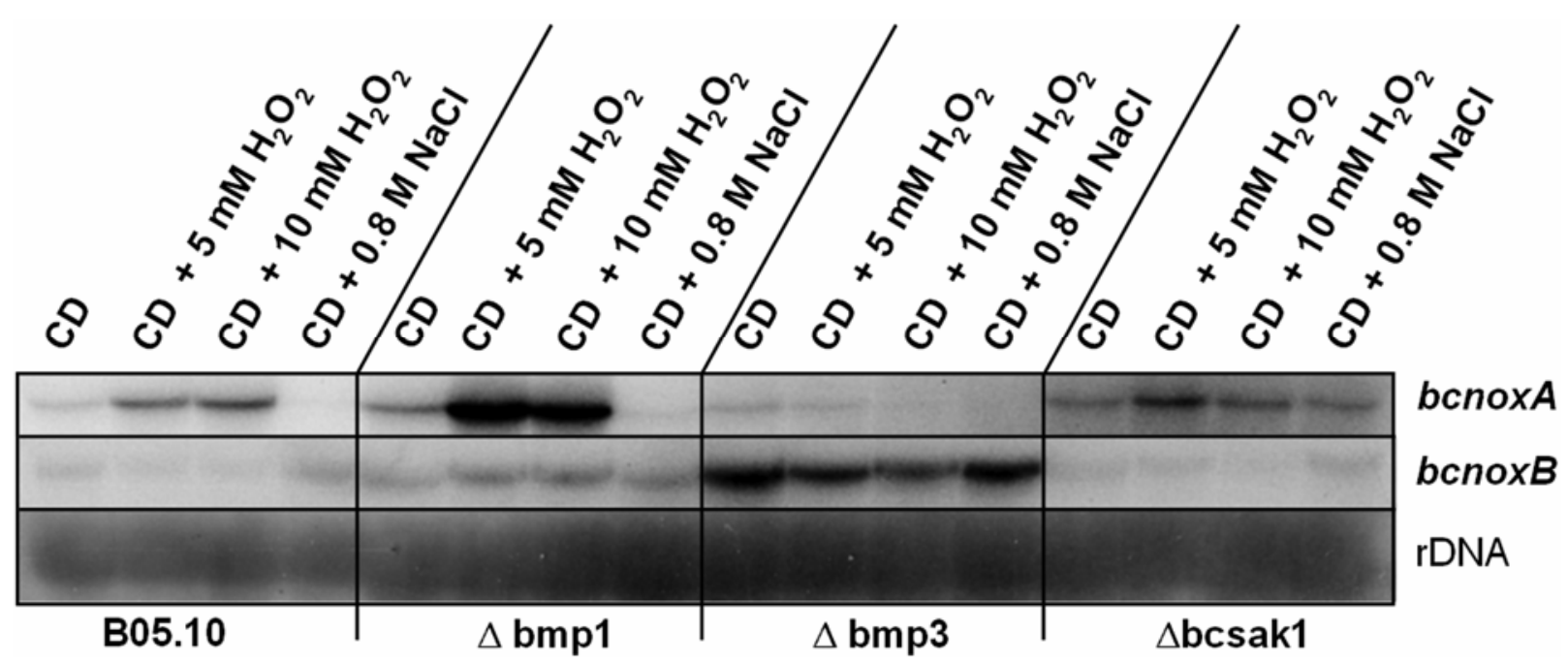

Fig. 3. Expression of bcnoxA and bcnoxB in three MAPK mutants of Botrytis cinerea and the wild-type strain. The wild type and the mutants were incubated in $1.5 \%$ malt medium for $24 \mathrm{~h}$ and transferred to Czapek Dox (CD) medium, pH 5.2, for $24 \mathrm{~h}$. Subsequently, mycelia were shifted in CD medium with different stress conditions $\left(5 \mathrm{mM}\right.$ and $10 \mathrm{mM} \mathrm{H}_{2} \mathrm{O}_{2}$ and $\left.0.8 \mathrm{M} \mathrm{NaCl}\right)$. RNA was isolated and used for a Northern hybridization with bcnoxA or bcnoxB probes, respectively. As a loading control, the blot was hybridized with a $B$. cinerea rDNA probe. The data are representative for three biological repeats. 
spore isolation. Homokaryotic derivatives have been obtained from all five primary transformants showing only the diagnostic fragment and lacking the wild-type fragment (data not shown) and were named $\Delta$ bcnoxA-4, $\Delta$ bcnoxA-5, $\Delta$ bcnoxA16, $\Delta$ bcnoxA-19, and $\triangle$ bcnoxA-20. The PCR data were confirmed by Southern analyses (Fig. 4B): the EcoRI-digested total DNA of the five mutants and the recipient strain B05.10 (wild type) were blotted and hybridized with the labeled $3^{\prime}$ flank. Whereas the wild type showed a hybridizing band of approximately $3.15 \mathrm{~kb}$, in the five mutants, this fragment was replaced by a $2.17-\mathrm{kb}$ fragment as, expected.

For the replacement of bcnoxB, the knock-out vector $\mathrm{p} \triangle \mathrm{NOXB}$ was created (described below). The replacement construct was excised with $K p n I$ and EcoRI and used to transform strain B05.10. Hygromycin-resistant transformants were tested by PCR for homologous integration. The primer pair 1/11 (binding within the resistance cassette and outside the replacement fragment, respectively) (Supplementary Fig. S1A) yielded a fragment of approximately $1.8 \mathrm{~kb}$ only if the fragment had been integrated by double crossover at the homologous site. Of the 12 transformants obtained, 6 showed this diagnostic band and all were heterokaryons, because PCR with primer pair 12/13 yielded the fragment specific to the wild type $(1,195 \mathrm{bp})$. Six of the primary transformants were subjected to one round of single-spore isolation. Homokaryotic derivatives showing only the diagnostic fragment and lacking the wild-type fragment have been obtained from all six primary transformants (data not shown). They were named $\Delta$ bcnoxB-1, $\Delta$ bcnoxB-3, $\Delta$ bcnoxB-4, $\Delta$ bcnoxB-5, $\Delta$ bcnoxB-6, and $\triangle$ bcnoxB-7. The PCR data were confirmed by Southern analyses: BamHI-digested total DNA of seven mutants, including one that did not show the diagnostic band $(\Delta \mathrm{bcnoxB}-2)$ in the PCR reaction and the recipient strain $\mathrm{B} 05.10$ (wild type), were blotted and hybridized with the labeled $3^{\prime}$ flank. Whereas the wild type showed a hybridizing band of more than $10 \mathrm{~kb}$, in six of the seven mutants, this fragment was replaced by an 8$\mathrm{kb}$ fragment as expected. $\Delta$ bcnoxB-4, $\Delta$ bcnoxB-5, and $\Delta$ bcnoxB-6 showed additional ectopic integrations whereas, in the genome of $\Delta \mathrm{bcnoxB}-2$, the replacement fragment has integrated ectopically. This transformant showed the wild-type fragment and probably a second hybridizing fragment with similar size. Transformants $\Delta$ bcnoxB-1, $\Delta$ bcnoxB-3, and $\Delta$ bcnoxB-7 showed only the replacement fragment of approximately $8 \mathrm{~kb}$ and were used for further analyses. $\triangle$ bcnoxB-3 was used as recipient for transformation with the bcnoxA replacement construct to yield the double knock-out mutants $\Delta$ bcnoxAB-1 and $\Delta$ bcnoxAB-2 (described below).

\section{Characterization of $\Delta$ bcnox mutants in vitro.}

Double and single mutants of the bcnox genes showed only a slight reduction in growth rate compared with the wild type on complete medium (CM) (Fig. 5). There was no difference in colony morphology on CM, in sporulation, or in the germination rate of conidia (data not shown).

To test whether deletion of the two NADPH oxidase genes affects growth during different stress conditions, the mutants were grown on $\mathrm{CM}$ under oxidative stress induced by menadione $(500 \mu \mathrm{M})$ and $\mathrm{H}_{2} \mathrm{O}_{2}(2 \mathrm{mM}$ and $5 \mathrm{mM}$, respectively) and osmotic stress $(0.8 \mathrm{M} \mathrm{NaCl})$ for 3 days (Fig. 5). All the mutants showed a slight reduction in growth on media containing $500 \mu \mathrm{M}$ menadione compared with the wild type. A further reduction in growth was observed during oxidative
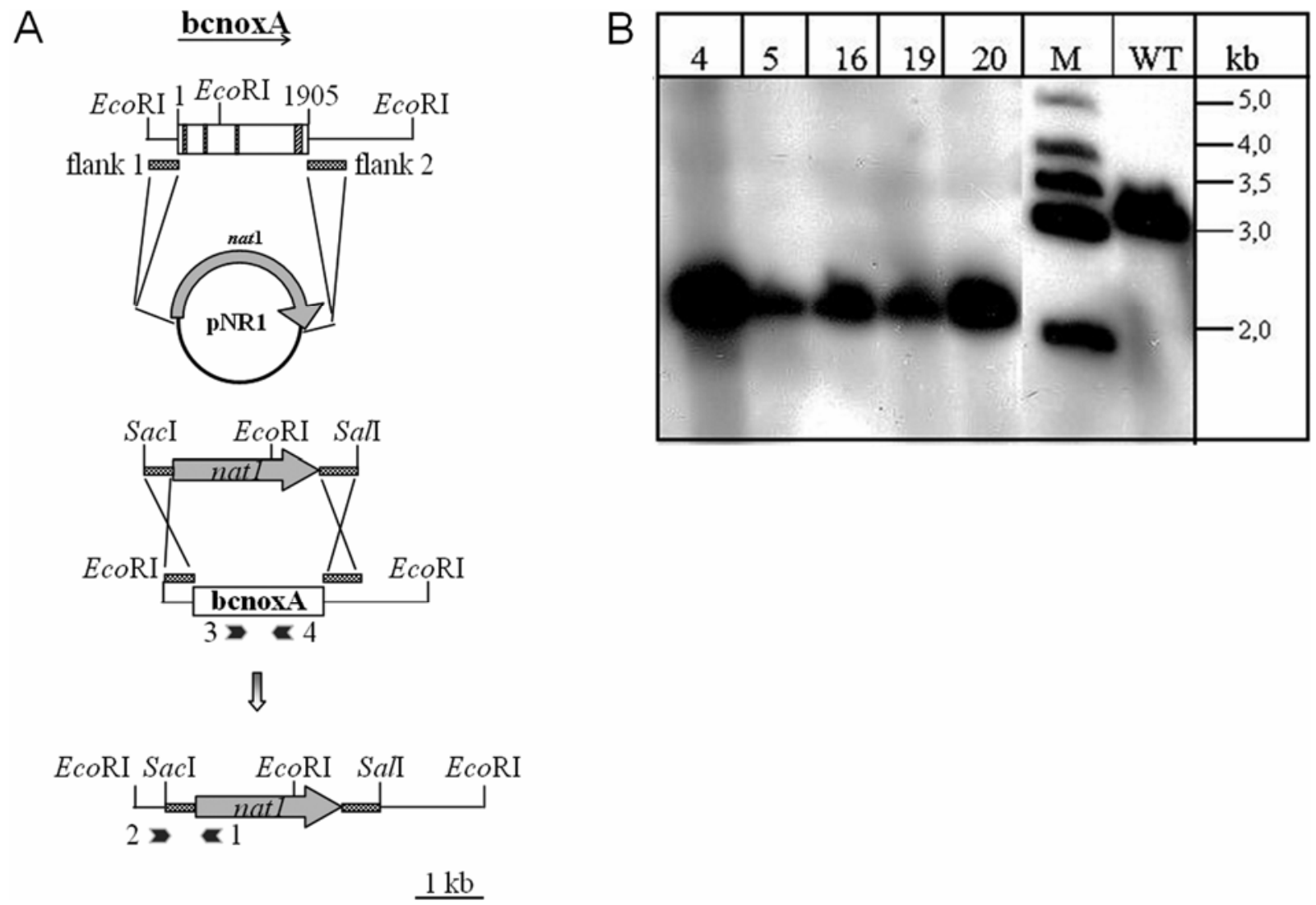

Fig. 4. Generation of $\Delta$ bcnoxA mutants. A, Gene replacement strategy for bcnoxA. The coding region is boxed; an arrow indicates orientation. Vector part of pNR1: thick black line; nourseothricin resistance cassette (nat1): gray. Primer binding sites are indicated by arrowheads. B, Southern analysis of transformants. Genomic DNA preparations of the putative deletion mutants ( $\Delta$ bcnoxA-4, $\Delta$ bcnoxA-5, $\Delta$ bcnoxA-16, $\Delta$ bcnoxA-19, and $\Delta$ bcnoxA-20) and the wildtype B05.10 (WT) were digested with EcoRI, separated in an agarose gel, blotted on a nitrocellulose membrane, and hybridized to the 3' flank of bcnoxA. M: molecular weight marker. 
stress mediated by $\mathrm{H}_{2} \mathrm{O}_{2} . \Delta$ bcnoxA and $\Delta$ bcnoxB both showed significant reduction in growth rate on 2 and $5 \mathrm{mM} \mathrm{H}_{2} \mathrm{O}_{2}$; the double knock-out mutant $\triangle$ bcnoxAB showed an additive effect. Osmotic stress mediated by $0.8 \mathrm{M} \mathrm{NaCl}$ had a slight effect on growth of $\triangle$ bcnoxA (and $\triangle$ bcnoxAB) mutants, but not on $\triangle$ bcnoxB. Obviously, both NADPH oxidases are necessary for normal tolerance to oxidative stress inflicted by $\mathrm{H}_{2} \mathrm{O}_{2}$, a rather unexpected result, because both are involved in ROS generation!

\section{BcnoxA and BcnoxB have impact on sclerotia formation.}

Because of the role of NADPH oxidases in P. anserina and A. nidulans in sexual development, single and double mutants of bcnoxA and bcnoxB were analyzed for their sexual reproduction. A prerequisite for sexual reproduction in $B$. cinerea is the formation of sclerotia, which-after fertilization with microconida-differentiate apothecia (Williamson et al. 2007). The mutants were incubated on CM for 6 weeks to induce formation of sclerotia and microconidia. In contrast to the wild type, $\triangle$ bcnoxA and $\triangle$ bcnoxAB mutants never showed any de- velopment of sclerotia, whereas one of the three $\Delta$ bcnoxB mutants tested ( $\Delta$ noxB-3) formed a few sclerotia only which, in addition, were small and of abnormal appearance (data not shown). To substantiate that BcnoxA and BcnoxB both are necessary for sclerotia development, inhibitor studies were performed. The addition of non-growth inhibiting concentrations of the Nox inhibitor diphenyleneiodonium (DPI) fully inhibited formation of sclerotia in the wild type (Fig. 6).

With the above-mentioned exception, the bcnox mutants produced no sclerotia; therefore, female sexual development (apothecia and ascospore formation) could not be monitored. To test whether the bcnox mutations had any effect on male fertility, crosses were performed using strain SAS405 (MAT1-2) as the female partner (strain B05.10, the genetic background of the bcnox mutants, carries MAT1-1). Microconidia of the wild type and $\triangle$ bcnoxA, $\triangle$ bcnoxB, and $\triangle$ bcnoxAB mutants were used for fertilization of sclerotia of SAS405. All crosses yielded mature apothecia and fully developed asci. The germination rate of ascospores was comparable in all crosses, regardless of whether the male parent was a wild type or a bcnox

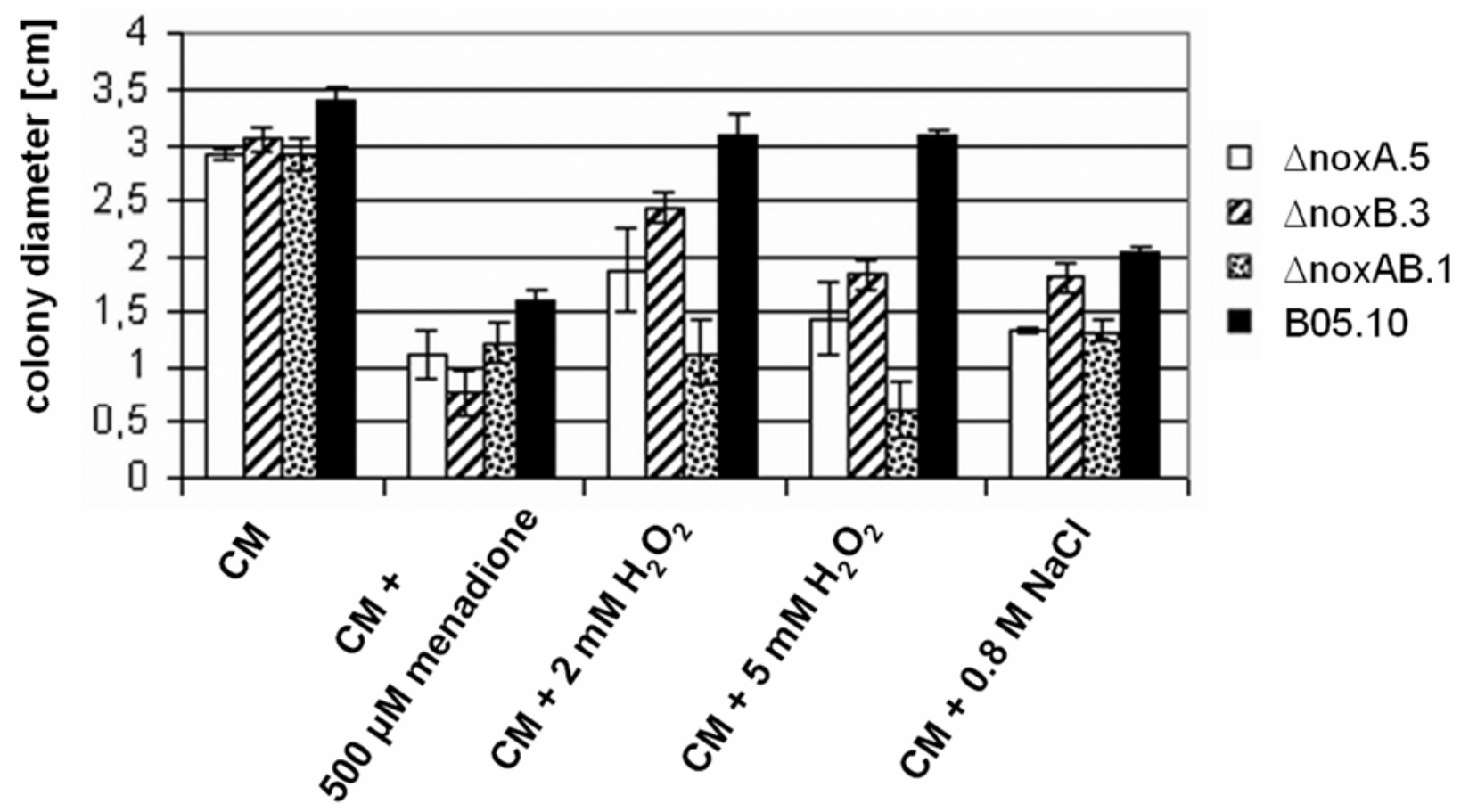

Fig. 5. Colony diameters of $\Delta$ bcnox mutants and wild-type strain B 05.10 after 3 days on complete medium (CM) containing different stressors as indicated below the graphs. Values are averages from at least five colonies, bars indicate standard deviation.

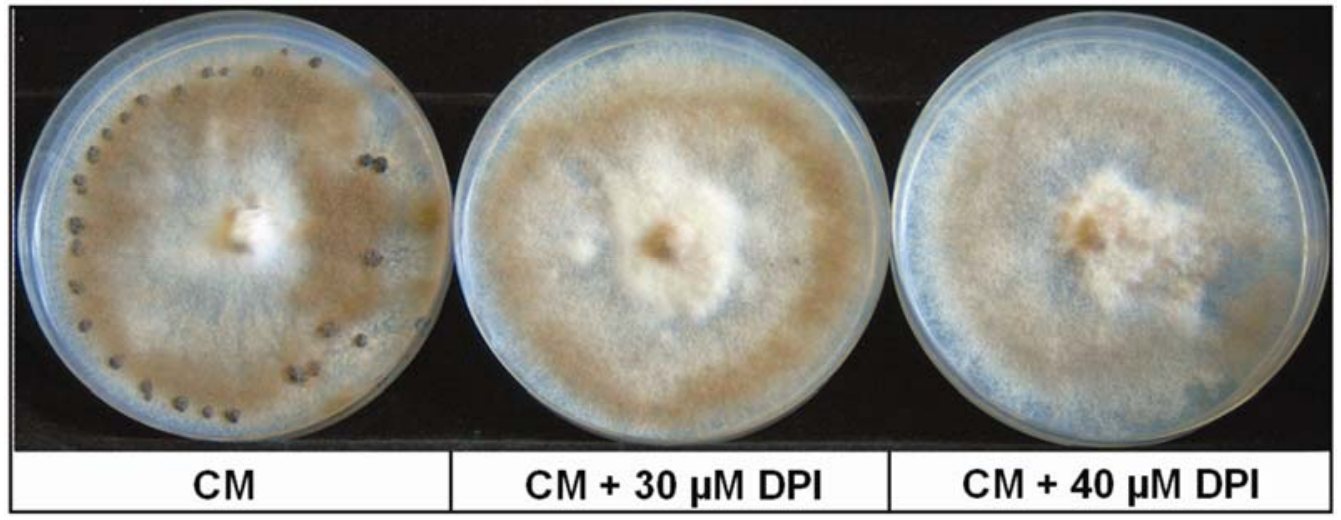

Fig. 6. Impact of diphenyleneiodonium (DPI) on sclerotia formation in the wild-type strain B05.10, incubated for 6 weeks at $21^{\circ} \mathrm{C}$ in darkness on complete medium with or without DPI ( 30 or $40 \mu \mathrm{M}$, as indicated). 
mutant. A test for germination on media supplemented with nourseothricin or hygromycin confirmed a normal segregation pattern for the resistance markers, indicating that none of the meiotic progeny displayed viability problems.

In conclusion, Bcnox $\mathrm{A}$ and $\mathrm{Bcnox} B$ have a strong impact on sclerotial development but not on male fertility, ascospore germination, and meiotic segregation.

BcnoxA and BcnoxB have impact on pathogenicity.

To analyze the effect on virulence of both NADPH oxidases, primary leaves of young bean plants were infected with conidial suspensions of $\triangle \mathrm{bcnox} A, \triangle \mathrm{bcnoxB}$, and $\triangle \mathrm{bcnox} A B$ mutants and the wild-type strain. Lesion sizes were determined 2 and 3 days postinoculation (dpi). In the wild-type control, small primary necrotic lesions were formed $2 \mathrm{dpi}$, which turned into secondary (spreading) lesions with a diameter of approximately $1.4 \mathrm{~cm}$ (3 dpi). All single mutants were able to infect bean tissue and to form primary and secondary lesions. However, $\Delta$ bcnoxB mutants were strongly delayed in primary lesion formation whereas $\Delta$ bcnoxA mutants showed normal primary lesion formation but slower expansion of secondary lesions. For both mutants, lesion diameters were smaller at 3 dpi compared with the wild type (Fig. 7). The double knock-out mutants showed an additive effect: formation of primary lesions occurred only 3 dpi and formation of spreading lesions was strongly impaired. Both NADPH oxidases are necessary for full pathogenicity in $B$. cinerea, and they have complementary functions. To obtain detailed information about the virulence defect, bcnox mutants and the wild type were analyzed for their ability to penetrate on onion epidermal cells. Onion epidermis was inoculated with spores of the mutants and the wild type. The samples were analyzed microscopically $24 \mathrm{~h}$ postinoculation (hpi) after staining with Lactophenol Blue. Only fungal structures outside the epidermal cells are stained when using this method. Conidia of the wild type and the bcnoxA mutants penetrated the onion epidermal cells directly after germination with the formation of appressoria-like structures (Fig. 8). The $\triangle$ bcnoxB and $\triangle \mathrm{bcnox} A B$ mutants were unable to penetrate the epidermal cells within this period, even though growth of the mutants on the surface of the onion epidermis was observed (Fig. 8). These observations suggest that the retarded formation of primary lesions by bcnoxB mutants is due to an impaired formation of penetration structures, whereas bcnoxA mutants are able to penetrate host tissue to the same extent as the wild type.

To confirm the correlation between the phenotype of the $\triangle \mathrm{bcnox} A$ and $\Delta \mathrm{bcnoxB}$ mutants and the inactivation of the genes, a complementation was performed. Intact copies of both genes, including $1 \mathrm{~kb}$ of the promoter and terminator regions, respectively, were cloned into the hygromycin-resistance vector pOLIHP for bcnoxA and into the nourseothricinresistance vector pNR1 for bcnoxB. The linearized complementation fragments were transformed into the $\Delta$ bcnoxA-5 mutant and the $\Delta$ bcnoxB-3 mutant. Colonies that were resistant to hygromycin and nourseothricin were isolated and analyzed for complete integration of the fragments. Two bcnoxA transformants and three bcnox $B$ transformants contained the full wild-type gene copy (tested by PCR and Southern analy-

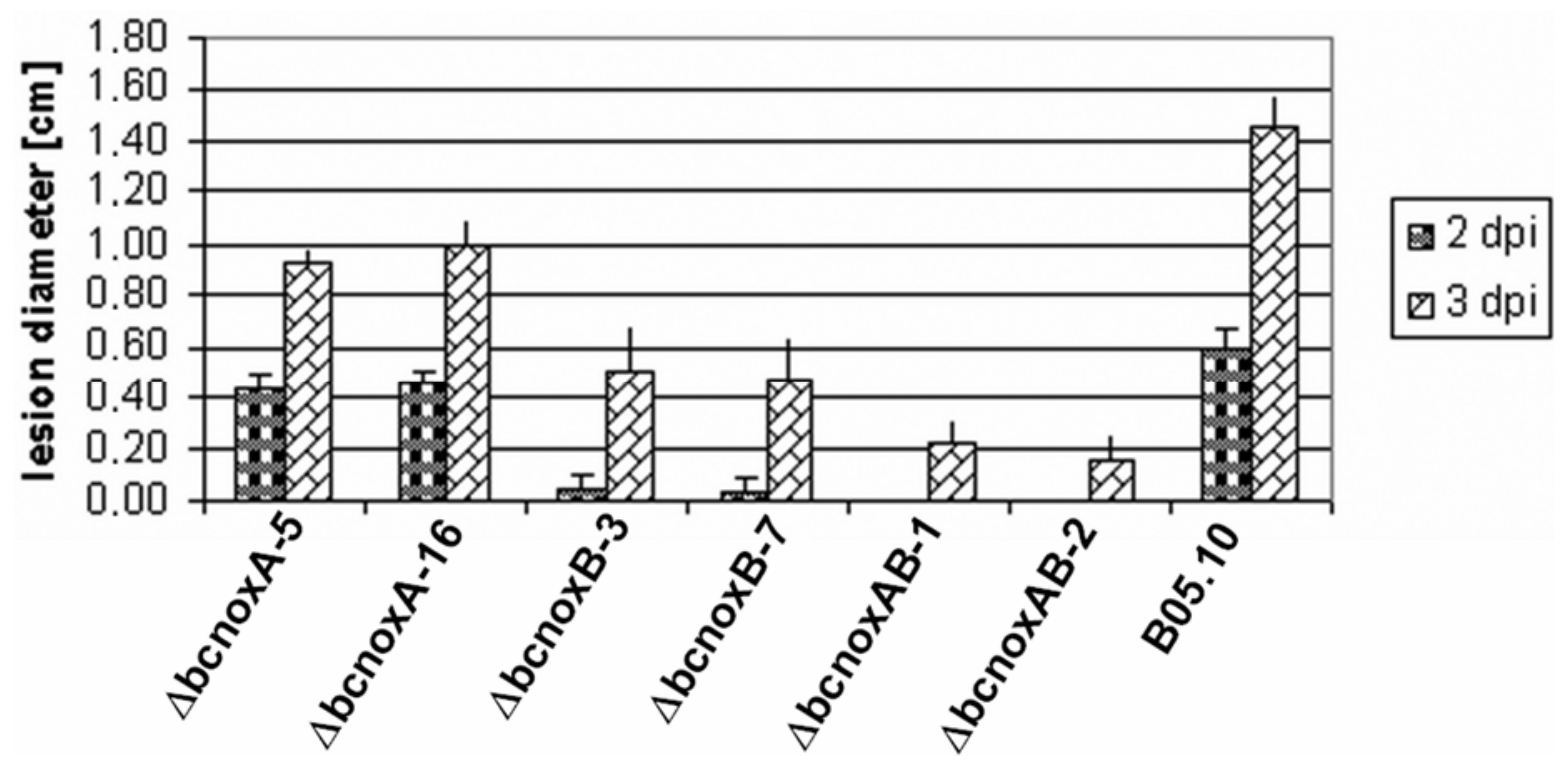

Fig. 7. Pathogenicity test on bean leaves. Compilation of infection tests, using three different $\Delta$ bcnox mutants. Values are averages from at least eight lesions each (from two different plants).

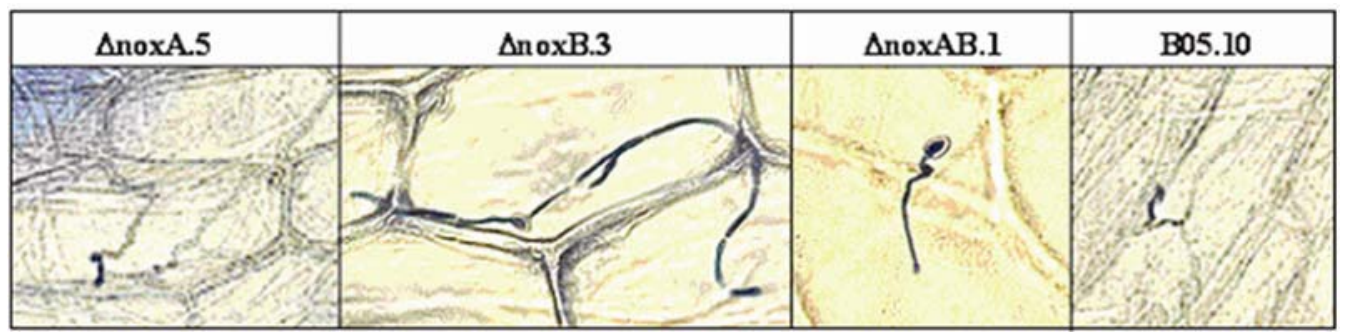

Fig. 8. Penetration assay of $\Delta$ bcnox mutants. Comparison of penetration structures of the wild type and $\Delta$ bcnox mutants on epidermal cells of onion $24 \mathrm{~h}$ postinoculation. 
ses) (data not shown). These complemented transformants were able to infect plant tissue at the same time and rate as the wild type, and they also had increased resistance against oxidative stress (data not shown).

\section{The $\Delta$ bcnox mutants are not significantly impaired in $\operatorname{ROS}$ production.}

The production of $\mathrm{H}_{2} \mathrm{O}_{2}$ by $\mathrm{B}$. cinerea within the host tissue was detected cytochemically using electron microscopy to locate the deposition of electron-dense cerium perhydroxides, formed by the reaction of cerium ions with $\mathrm{H}_{2} \mathrm{O}_{2}$ (Bestwick et al. 1997; Briggs et al. 1975; Shinogi et al. 2003). Bean plants were infected with conidial suspension of wild-type B05.10 and mutants $\Delta$ noxA-5, $\Delta$ noxB-3, and $\Delta$ noxAB-1. Samples were taken 24 and 48 hpi, fixed, and analyzed. No difference was observed between the wild type and the mutant strains in the density of deposits. The wild type and the $\Delta$ bcnox mutants showed no detectable difference in formation of $\mathrm{H}_{2} \mathrm{O}_{2}$ in the early stages of infection in the samples taken $24 \mathrm{hpi}$. In the control experiment that lacked cerium chloride, a deposition of electron-dense material was not observed, proving the specificity of $\mathrm{H}_{2} \mathrm{O}_{2}$ detection (data not shown).

Additional experiments for detection of $\mathrm{O}_{2}^{-}$were done with onion epidermal cells inoculated with the mutant strains and the wild type. Samples were incubated with nitroblue tetrazolium (NBT), which forms a dark-blue water-insoluble formazan precipitate upon reduction by superoxide radicals. Blue precipitate was detected in the cell wall of germinated conidia, in the appressoria-like structures, and in hyphae growing inside the onion epidermal cells. There was no significant difference in precipitate formation between the mutants and the wild-type strain (data not shown), suggesting that an alternative source of ROS is involved in their production during plant infection. This interpretation was corroborated by incubating the samples with $50 \mu \mathrm{M}$ DPI, an inhibitor of NADPH oxidases, prior to the staining with NBT. In these samples, blue formazan precipitation was detected in conidia and mycelium growing on onion epidermal cells. Also, no difference in formation of blue formazan was observed between the $\Delta$ bcnox mutants and the wild type in hyphae grown in axenic cultures (data not shown).

\section{Bcnox $R$ is involved in activation of $B c n o x A$ and $B c n o x B$.}

Recently, Takemoto and associates (2006) presented evidence that a fungal (partial) homolog (NoxR) of the regulatory subunit $\mathrm{p} 67^{\text {phox }}$ of the mammalian Nox complex is involved in regulation of NoxA activity in the endophyte E. festucae: a $\Delta$ noxR mutant had a phenotype similar to a $\Delta$ noxA mutant. Because the genome sequence of $B$. cinerea contained a noxR homolog (BC1G_06200.1, supercontig 29), we carried out a functional analysis of this gene to investigate its role in the Nox complex in $B$. cinerea. The genomic region containing the ORF BC1G_06200 was sequenced again because the BROAD genome sequence is of limited quality. Putative introns were confirmed by RT-PCR analyses (data not shown). The corrected sequence comprises an ORF of $1,915 \mathrm{bp}$, interrupted by five introns, and encodes a polypeptide of 541 amino acids showing high homology to NoxR of E. festucae; hence, the gene was named bcnoxR. Like NoxR of E. festucae, BcNoxR shows only partial homology to mammalian p67 $7^{\text {phox }}$. Whereas the N-terminal domain structure is similar (four tetratricopeptide repeat motifs for Rac binding; a putative Nox activation domain), the C-terminus lacks domains which are involved in the interaction with other regulatory domains of the mammalian Nox complex (e.g., the SH3 domain) (Bedard and Krause 2007; Lambeth 2004). However, like NoxR of E. festucae, BcnoxR contains a phox/Bem1p domain (Takemoto et al.
2006); thus, the fungal Nox complex probably differs from that of mammals (Takemoto et al. 2007). Using an approach similar to that described above for bcnoxA and bcnoxB, a gene replacement construct was designed (below and Supplementary Fig. S2). Altogether, five homokaryotic $\Delta$ bcnoxR mutants were obtained (below) and subjected to the same experiments as the bcnoxA and bcnoxB mutants. Three of the $\Delta$ bcnoxR mutants have the same growth characteristics as the double mutant $\triangle$ bcnoxAB (Fig. 9). They show a slightly reduced growth rate on $\mathrm{CM}$ and have the same strong $\mathrm{H}_{2} \mathrm{O}_{2}$ sensitivity as the double mutants. The $\Delta$ bcnoxR mutants also are unable to form sclerotia (data not shown). In a pathogenicity test on bean plants, the $\Delta$ bcnoxR mutants showed a virulence defect indistinguishable from $\triangle$ bcnoxAB mutants (Fig. 10). $\Delta$ bcnoxR mutants showed a severely retarded formation of primary lesions and a significant reduction in the capacity to form spreading lesions. A comparison of infected bean leaves 3 dpi is shown in Figure 10; the detailed values including the results for 2 dpi are presented in Supplementary Figure S3. In an onion epidermis penetration assay, the $\Delta$ bcnoxR mutants had a phenotype identical to that of $\triangle$ bcnoxAB double mutants (data not shown). In conclusion, a mutant lacking the putative regulatory subunit BcnoxR mimics the phenotype of mutants that are deleted in both the NoxA and the NoxB catalytic subunits of the NADPH oxidase complexes.

\section{DISCUSSION}

NADPH oxidases are key enzymes in defense reactions against pathogens in higher eukaryotes. However, their range of functions is much broader: they are involved in various biological functions, such as regulation of cell proliferation in mammals (Arnold et al. 2001; Suh et al. 1999) and root hair extension in plants (Foreman et al. 2003). In filamentous fungi, homologs of NADPH oxidases are involved in a wide variety of differentiation functions, including sexual reproduction, formation of penetration structures, and establishment of mutualistic interactions (Egan et al. 2007; Lara-Ortiz et al. 2003; Malagnac et al. 2004; Tanaka et al. 2006).

Here, the role of two NADPH oxidases in the necrotrophic phytopathogen $B$. cinerea was investigated. Single and double knock-out mutants of both NADPH oxidase genes in $B$. cinerea showed that BcnoxA and BcnoxB are essential for the formation of sclerotia and have great impact on pathogenic development.

Sclerotia are organs that permit survival of the fungus during unfavorable environmental conditions, and they are essential for sexual reproduction during development of apothecia. $\triangle$ bcnoxA (and $\triangle \mathrm{bcnox} A B$ ) mutants failed to produce any sclerotia, as did $\Delta$ bcnoxB mutants in most cases. A direct causal correlation between the lack of sclerotia and the loss of NADPH oxidase activity was substantiated by incubating the wild-type B05.10 with the Nox inhibitor DPI which fully abolished formation of sclerotia. Likewise, deletion of the putative Nox regulator BcNoxR led to the inability to form sclerotia. Cross fertilization experiments showed that $\Delta$ bcnoxA and $\Delta$ bcnoxB mutants were not disturbed in male fertility. They showed normal formation of microconidia, and crosses with a wild-type female partner yielded normal apothecia with mature asci. Ascospores collected from such crosses showed no defect in germination and normal segregation patterns were found for the antibiotic resistance genes.

The lack of sclerotia formation in the $\Delta$ bcnox mutants does not allow conclusions on their impact on female fertility. In contrast to $P$. anserina, ascospore germination is not affected in any of the mutants. Also, the effects observed in vegetative mycelia (lack of aerial hyphae and pigmentation, and so on) in 
$P$. anserina were not observed in $B$. cinerea: all $\Delta$ bcnox mutants showed normal colony morphology, sporulation, and conidial germination. Thus, the Nox enzymes have a different impact on vegetative differentiation processes in the fungi analyzed so far.

Interestingly, the NADPH oxidase mutants of $B$. cinerea show sensitivity to oxidative stress. Whereas sensitivity to menadione, an $\mathrm{O}_{2}^{-}$-generating agent, is not particularly evident, sensitivity to $\mathrm{H}_{2} \mathrm{O}_{2}$ seems to be very strong. The additive effect observed during $\mathrm{H}_{2} \mathrm{O}_{2}$ treatment in the double mutant suggests that $\mathrm{Bcnox} \mathrm{A}$ and $\mathrm{Bcnox} \mathrm{B}$ function independently during oxidative stress. A possible explanation for this sensitivity could be the role of NADPH oxidases in oxygen sensing in the way postulated by Geiszt and associates (2000). A sensory function would lead to a deregulation of oxidative stress-dependent signaling pathways in the mutants and to a failure to induce or activate scavenging enzymes. Increased sensitivity of $\triangle$ bcnoxA mutants to oxidative stress is in agreement with the gene expression patterns. Transcript levels of bcnoxA were increased during oxidative stress mediated by $\mathrm{H}_{2} \mathrm{O}_{2}$, as also was observed in $C$. purpurea (Giesbert et al. in press). Expression studies using MAPK mutants of $B$. cinerea showed that, in contrast to $A$. nidulans, the stress-activated kinase Bcsak1 (Segmüller et al. 2007) is not involved in bcnoxA regulation. BcnoxA was negatively modulated by the MAPK Bmp1 (stronger induction under oxidative stress in $\Delta$ bmp1) and positively by Bmp3 (no induction in $\Delta \mathrm{bmp} 3$ ).

Bmp3 also has a strong influence on bcnoxB expression because the gene is highly deregulated in the $\Delta$ bmp3 mutant. Whereas the gene shows no significant differences in expression patterns in the wild type, $\Delta \mathrm{bmp} 1$, or $\Delta$ bcsak 1 , the bcnox $B$ transcript level in the $\Delta$ bmp3 mutant was very high during all conditions tested. Therefore, Bmp3 is likely the major regulator of both bcnox genes. Interestingly, $\Delta \mathrm{bmp} 3$ is-like $\triangle$ bcnoxA mutants-impaired in the formation of sclerotia (Rui and Hahn 2007), consistent with a role of Bmp3 in positive regulation of bcnoxA.
Bmp3 is homologous to the "cell-wall integrity" MAPK Slt2 in yeast. Because of the involvement of ROS in cell-wall biosynthesis and differentiation (Takemoto et al. 2007), it seems probable that the bcnox genes are controlled mainly by this pathway and not by the SAK pathway, as is the bcnoxA ortholog in A. nidulans. However, the B. cinerea SAK cascade also is involved in differentiation: $\Delta$ bcsak mutants produce no conidia and profuse numbers of sclerotia, and they cannot penetrate plant tissue (Segmüller et al. 2007). Therefore, there must be considerable cross-talk between these MAPK cascades. The observed sensitivity of the $\Delta$ bcnox mutants to oxidative stress was unexpected because the enzymes themselves contribute to ROS production. Also, in $C$. purpure, $\Delta$ cpnox $1 \mathrm{mu}-$ tants were more sensitive to menadione (Giesbert et al. in press). However, the induction of bcnoxA transcription by oxidative stress points to the same direction. Obviously, the mechanisms of ROS homeostasis are more complex than anticipated. Several explanations are possible; because the major function of Nox enzymes is to deliver $\mathrm{O}_{2}^{-}$in a precisely defined matter with regard to time and place for differentiation of cell wall structures, it could be speculated that modifications of the cell wall are involved in resistance against oxidative stress. A biological function for NADPH oxidases in $B$. cinerea could be that the oxidative stress encountered in planta induces Noxcatalyzed differentiation processes which are needed for penetration or colonization.

The failure to detect differences in ROS status in vegetative hyphae and in planta between the wild type and any of the mutants demonstrates that the Nox enzymes are not major players in the secretion of ROS, though detection of ROS differences are dependent on the balance of ROS synthesis and breakdown and, hence, they might be revealed only at certain stages of development. Nevertheless, there must be alternative sources of ROS which are responsible for this fungal oxidative attack. The observations in P. anserina (Malagnac et al. 2004) and $M$. grisea (Egan et al. 2007) confirm this idea: in both cases, deletion of nox genes even led to increased overall ROS produc-

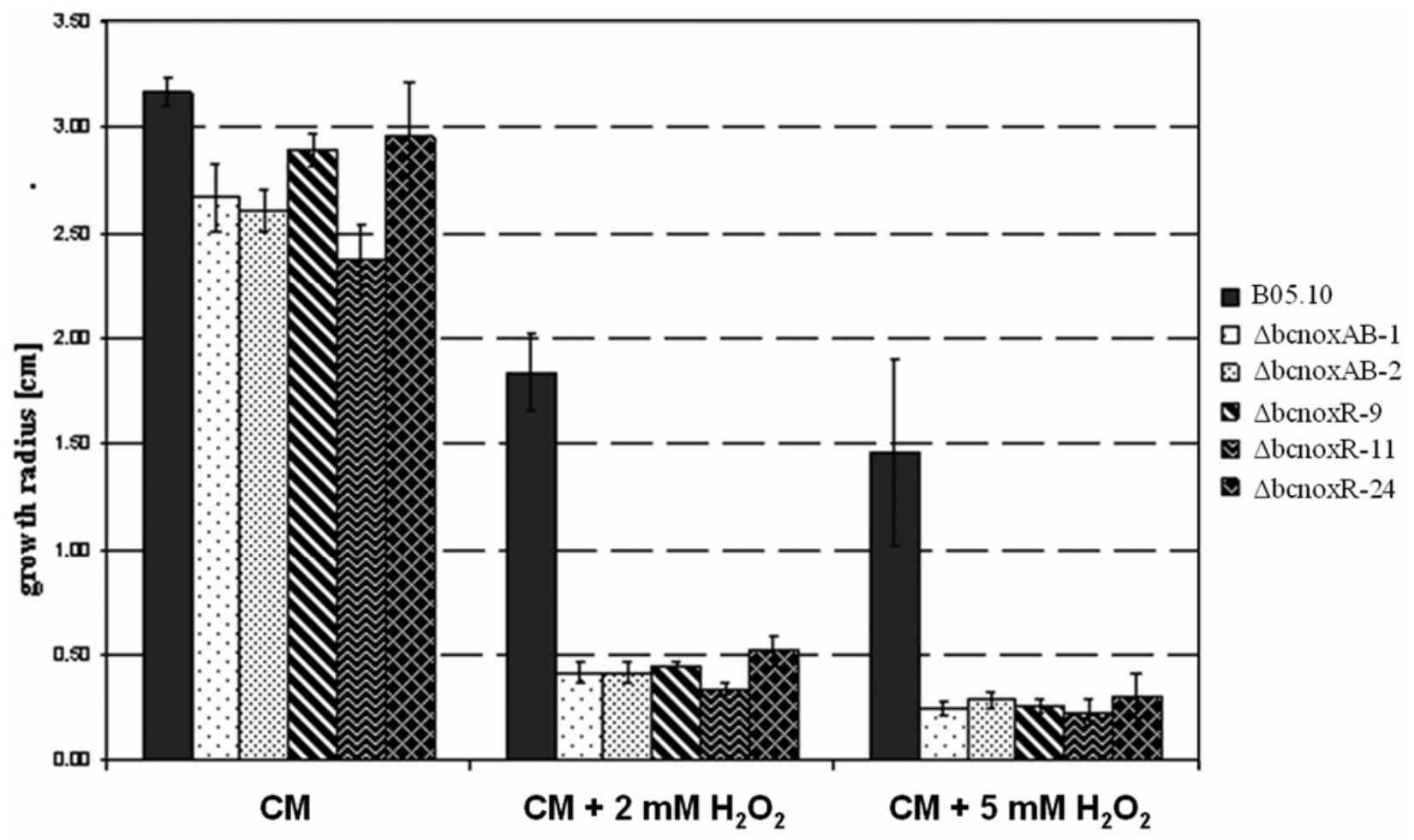

Fig. 9. Colony diameters of $\Delta b c n o x R$ and $\Delta b c n o x A B$ mutants under oxidative stress (values are averages of at least five colonies). 
tion. In M. grisea, a third putative nox gene is present, noxC, which could be responsible for this effect. However, the genome of $B$. cinerea obviously does not contain a third nox gene. Only in E. festucae is there direct evidence that secreted $\mathrm{H}_{2} \mathrm{O}_{2}$, present as a layer around the endophytic hyphae, is lost in the $\Delta$ noxA mutant.

Taken together, the available data strongly suggest that Nox enzymes in fungi are involved mainly in differentiation processes, probably through their role in cell wall biogenesis. In the fungi studied so far, the particular roles of the individual NADPH oxidases are diverse. In $B$. cinerea, the NADPH oxidases both are required for differentiation of sclerotia, whereas their role in virulence is different: $\mathrm{BcnoxB}$ is important for penetration while BcnoxA is involved in spreading of lesions. These different roles also are reflected by their specific regulation pattern.

The structure of the fungal Nox complex concerning the regulatory subunits (which are well known for vertebrate NADPH oxidases) is not yet clear; thus far, only (partial) homologs of the regulatory subunit $\mathrm{p} 67^{\text {phox }}$ have been detected in the available genome sequences. Only one p67 $7^{\mathrm{phox}}$-like protein has been investigated in detail: in E. festucae, NoxR was shown to be necessary for NoxA activity (though, interestingly, only in planta!). NoxR lacks several domains which are known to be involved in the mammalian complex in interaction with other regulatory subunits. Because orthologs of these subunits (e.g., p40 phox and $\mathrm{p} 47^{\text {phox }}$ ) have not been found so far in fungal genomics, the fungal Nox complex must have a different structure. BcnoxR of $B$. cinerea has high similarity to NoxR of E. festucae and the same overall structure. In contrast to E. festucae, however, BcnoxR is obviously important in vitro and in planta, and it is necessary for both catalytic subunits, $\mathrm{BcnoxA}$ and $\mathrm{B}$, because the $\Delta$ bcnoxR phenotype is identical to that of the $\triangle$ bcnoxAB double mutant. Again, it becomes obvious that the role of the Nox complex differs in fungi, and that it might be adapted to the specific life style: the role in the endophyte $E$. festucae is different from that in the hemibiotroph $M$. grisea and the necrotroph $B$. cinerea, making this ROS-generating complex a highly interesting object of "evo-devo" studies.

\section{MATERIALS AND METHODS}

\section{Fungal strains.}

B. cinerea Pers. (teleomorph Botryotinia fuckeliana (de Bary) Whetzel) B05.10 (Quidde et al. 1999) was used as recipient strain for the transformation experiments and as a wild-type control. For expression studies, mutant strains of B05.10 with
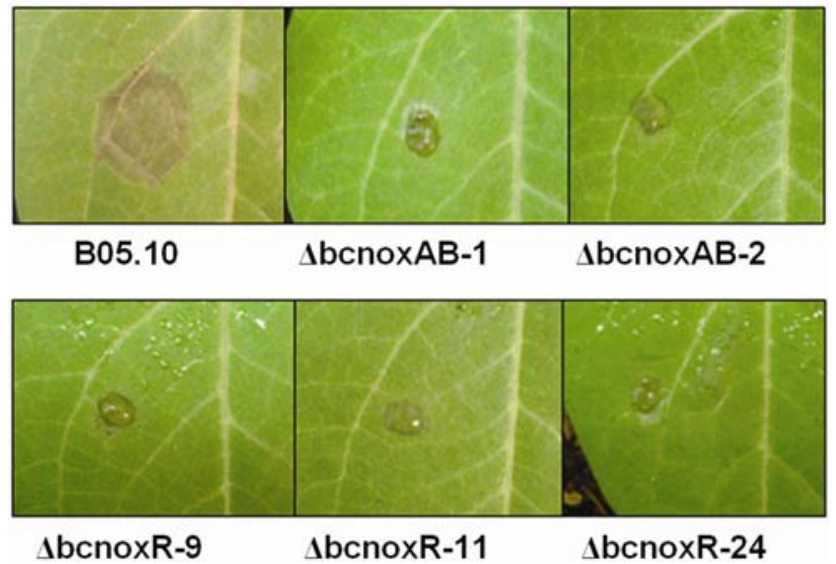

Fig. 10. Pathogenicity test on bean leaves: comparison of lesion diameters of $\Delta \mathrm{bcnoxR}$ and $\Delta \mathrm{bcnoxA} B$ mutants ( 3 days postinoculation). a deletion of the single MAPK-encoding genes $\triangle$ bcsak1 (Segmüller et al. 2007), $\Delta$ bmp1 (Doehlemann et al. 2006; Zheng et al. 2000) and $\Delta$ bmp3 (Rui and Hahn 2007) were used.

\section{Bacterial strains.}

For the propagation of lambda clones, strain LE $392 \mathrm{~F}^{-}$, hsdR574 (rk-mk+) supE4 lacY1 supE58 D [(lacIZY)6 galK2 galT22 metB1 trpR55 D (mrr-hsdRMS-mcrBC) (Stratagene, La Jolla, CA, U.S.A.) was used; subcloning was performed in strain TOP10F $^{-}\left(\right.$lacI $^{\mathrm{q}}$, Tu10 $\left(\right.$ Tet $\left.\left.^{\mathrm{r}}\right)\right]$, merA, $\Delta$ (mrr-hsdRMSmerBC), Ф80lac $2 \Delta \mathrm{M} 15, \Delta \mathrm{lacX} 74$, recA1, $\Delta$ araD139, $\Delta$ (araleu)7697, galU, galK, $\operatorname{rpsL}\left(\mathrm{Str}^{\mathrm{r}}\right)$, endA1, nupG (Invitrogen, San Diego, CA, U.S.A.).

\section{Media and culture conditions.}

B. cinerea strains were grown on CM (Pontecorvo et al. 1953 ) or potato dextrose agar (containing $10 \%$ bean leaves) at $20^{\circ} \mathrm{C}$ under near-UV for conidiation. Testing of stress sensitivity was performed by inoculating strains on $\mathrm{CM}$ plates supplemented with $\mathrm{H}_{2} \mathrm{O}_{2}$ (Merck, Poole, U.K.), menadione (Sigma, St. Louis), and $\mathrm{NaCl}$ (Merck) as indicated. Testing of NADPH inhibitor DPI (Sigma) was carried out on CM medium with a final concentration of $30 \mu \mathrm{M}$. Single conidial isolates were obtained by spreading $100 \mu \mathrm{l}$ of conidial suspension on Gamborg's B5 Medium (Duchefa Biochemie BV, Haarlem, The Netherlands) plates containing nourseothricin or hygromycin at $70 \mu \mathrm{g} \mathrm{ml}{ }^{-1}$. Conidia were germinated for $48 \mathrm{~h}$ and single colonies were transferred individually to new plates containing nourseothricin or hygromycin. For DNA isolation, mycelium was grown for 2 to 3 days at $20^{\circ} \mathrm{C}$ on $\mathrm{CM}$ agar with a Cellophane overlay. For RNA isolation, strains were cultivated in 300-ml Erlenmeyer flasks with 100 $\mathrm{ml}$ of a defined liquid $\mathrm{CD}$ medium containing $\mathrm{NaNO}_{3}$ at $1 \mathrm{~g}$ liter ${ }^{-1}$ instead of $3 \mathrm{~g} \mathrm{liter}^{-1}$ and glucose at $20 \mathrm{~g} \mathrm{liter}^{-1}$ instead of sucrose and $\mathrm{pH}$ 5.2. For oxidative and osmotic stress experiments, media were supplemented with menadione, $\mathrm{H}_{2} \mathrm{O}_{2}$ and $\mathrm{NaCl}$ as indicated. Stress mediated by $\mathrm{pH}$ was carried out in CD media (discussed above) with pH 7 to 9 . For temperature stress, fungi were incubated at $28^{\circ} \mathrm{C}$. Fungi were harvested after 2 days.

\section{Conidial germination tests.}

For analyses of conidiospore germination on glass surfaces in nutrient dependency (Doehlemann et al. 2005), spores were harvested from CM agar plates, filtrated over Nytex and suspended in $10 \mathrm{ml}$ of sterile water and washed three, times. Spore suspensions $(25 \mu \mathrm{l})$ with a concentration of $5 \times 10^{5}$ spores per milliliter were placed into the center of a cover slip and $475 \mu \mathrm{l}$ of B5 nutrient solution were added to the spores supplemented with fructose at a final concentration of 10.5 $\mathrm{mM}$. The cover slips were incubated in the dark at $20^{\circ} \mathrm{C}$ for several hours. Germination progress was monitored by light microscopy.

\section{Standard molecular methods.}

Fungal genomic DNA was isolated as described by Cenis (1992). Lambda DNA was isolated according to the standard method (Sambrook et al. 1989). Plasmid DNA was isolated using a plasmid DNA preparation kit (Genomed, Bad Oeynhausen, Germany).

\section{Southern blot analysis.}

Blots were performed according to Sambrook and associates (1989). Hybridization was carried out in $6 \times$ SSPE $(1 \times$ SSPE is $0.18 \mathrm{M} \mathrm{NaCl}, 10 \mathrm{mM}$ NaPO4, and $1 \mathrm{~nm}$ EDTA [pH 7.7]), 5× Denhardt's solution, $0.1 \%$ sodium dodecyl sulfate (SDS), and $50 \mathrm{mM}$ phosphate buffer, $\mathrm{pH} 6.6$, at $65^{\circ} \mathrm{C}$ for 16 to $20 \mathrm{~h}$ in the 
presence of a random-primed $\left[\alpha_{-}{ }^{32} \mathrm{P}\right] \mathrm{dCTP}$-labeled probe. Membranes were washed in $2 \times \mathrm{SSPE}$ and $0.1 \%$ SDS at $65^{\circ} \mathrm{C}$ before being exposed to an autoradiographic film.

\section{Northern analysis.}

RNA was isolated from mycelial samples using the RNAgents Total RNA Isolation System (Promega, Mannheim, Germany). Samples of 10 to $15 \mu \mathrm{g}$ of RNA were transferred onto Hybond- $\mathrm{N}^{+}$filters after electrophoresis on a $1 \%$ agarose gel containing formaldehyde according to Sambrook and associates (1989). Blot hybridizations were carried out in $0.6 \mathrm{M}$ $\mathrm{NaCl}, 0.16 \mathrm{M} \mathrm{Na}_{2} \mathrm{HPO}_{4}, 0.06 \mathrm{M}$ EDTA, $1 \% \mathrm{~N}$-lauroylsarcosine (Sigma), and 10\% dextran sulfate (Eppendorf AG, Hamburg, Germany), $\mathrm{pH}$ 6.5, as described for Southern blots.

\section{DNA sequencing.}

This was performed with an automatic sequencer LI-COR 4200 (MWG Biotech, Munich, Germany) using the Thermo Sequenase fluorescent-labeled primer cycle sequencing kit (Amersham Pharmacia, Piscataway, NJ, U.S.A.). For sequence analysis and construction of phylogenetic trees, the program DNA Star (Madison, WI, U.S.A.) was used.

\section{RT-PCR.}

For confirmation of introns in the bcnoxA gene, RT-PCR analyses were performed using the primers 20 and 21 (Table 1). For reverse transcription, $1 \mu \mathrm{g}$ of RNA was denatured with $200 \mathrm{ng}$ of primer 21 for $5 \mathrm{~min}$ at $70^{\circ} \mathrm{C}$ and then incubated with $500 \mathrm{nM}$ concentrations of deoxynucleoside triphosphates, $10 \mathrm{mM}$ concentration of dithiothreitol, and $200 \mathrm{U}$ of reverse transcriptase (SuperScript; Invitrogen) at $42^{\circ} \mathrm{C}$ for $1 \mathrm{~h}$. Subsequent PCRs contained $3 \mu \mathrm{l}$ of RT reaction, $50 \mathrm{ng}$ of each primer, $200 \mathrm{nM}$ concentrations of deoxynucleoside triphosphates, and $1 \mathrm{U}$ of Taq polymerase (BioTherm; GeneCraft, Lüdinghausen, Germany) and was carried out at $94^{\circ} \mathrm{C}$ for 4 min, followed by 35 cycles of $94^{\circ} \mathrm{C}$ for $1 \mathrm{~min}, 59^{\circ} \mathrm{C}$ for 1 min, and $70^{\circ} \mathrm{C}$ for $45 \mathrm{~s}$.

\section{Vector construction.}

A replacement vector for bcnoxA was designed as described in Figure 4A. By use of the primer pairs $5 / 6$ and $7 / 8$ (Table 1) the $5^{\prime}$ and $3^{\prime}$ flanking regions of the gene, respectively, were amplified. The flanking regions were cloned into vector pCR2.1. From this product, the $5^{\prime}$ and $3^{\prime}$ fragments were excised with $\mathrm{SacI} / \mathrm{XbaI}$ and ClaI/SalI, respectively, and cloned into the nourseothricin-resistance vector pNR1, as indicated in Figure 2a. The complete replacement fragment was excised with SacI/SalI and used to transform strain B05.10.

For complementation of bcnoxA in $\Delta$ bcnoxA-5, mutant $\Delta$ bcnoxA-5 was complemented using a $4.5-\mathrm{kb}$ fragment which was amplified by PCR using primer pair 9/10 (Table 1). The obtained fragment included the bcnoxA gene together with 1 $\mathrm{kb}$ each of the bcnoxA promoter and terminator. The sequence of the PCR product was determined to ensure correctness of the sequence. For construction of the complementation vector, the plasmid pOLiHP (Rolke et al. 2004) carrying the Escherichia coli hygromycin phosphotransferase gene $h p h$ under control of the $A$. nidulans oliC promoter and $\operatorname{trp} C$ terminator was used as a basic vector. The fragment was cloned in pCRBluntII-TOPO (Invitrogen), cut with SmaI, and cloned into the corresponding site of pOLiHP. By cutting with SacII, the complementation vector was linearized prior to transformation.

For construction of a replacement vector for $b c n o x B$, primer pairs 14/15 and 16/17 (Table 1) were used to amplify the 5' and $3^{\prime}$ flanking regions of the gene, respectively (Supplementary Figure. S1). The flanking regions were cloned into vector pCR2.1 excised with KpnI/SalI and HindIII/EcoRI, respectively, and cloned into the nourseothricin-resistance vector pOLiHP (Rolke et al. 2004), as indicated in Supplementary Figure. S1A. The replacement fragment was excised with KpnI/EcoRI and used to transform strain B05.10.

Complementation of bcnoxB in $\triangle$ bcnoxB.3 was performed by transformation of the mutant $\Delta$ bcnoxB-3 with a full-length copy of bcnoxB. A 4.3-kb fragment including bcnoxB together with $1 \mathrm{~kb}$ of the $5^{\prime}$ and $3^{\prime}$ noncoding sequences was amplified

Table 1. Polymerase chain reaction (PCR) primers used for construction of vectors, diagnostic and reverse-transcriptase PCR

\begin{tabular}{|c|c|c|}
\hline Number & Primer & Sequence \\
\hline 1 & OliCrev & 5'-TGT GAT GGA GTT GAG ATG GG-3' \\
\hline 2 & bcnox 2 Fl1_K F & 5'-CAT CCG AAT TCA TCG ATT TGC GCC C-3' \\
\hline 3 & bcNox $2 \_$CF1 & 5'-GGG TCC ACT CAA CTA TCT CCA TCG-3' \\
\hline 4 & bcNox2_CR1 & 5'-GCC TTG TAC TTC ATC GAT GGC TTT C-3' \\
\hline 5 & bcnox2_Fl1_F & 5'-CTG TAC GAG CTC TGG TTT CGA ACC-3' \\
\hline 6 & bcnox2_Fl1_R & 5'-CAG CAC CCA TCT TCT CTA GAT CC-3' \\
\hline 7 & bcnox 2 Fl2_F & 5'-GGG GGA ATA TCG ATA AGA ATG GGT GG-3' \\
\hline 8 & bcNox2_Fl2_R2 & 5'-GTT ATT GTC GAC AAA CTA GGG GGT GG-3' \\
\hline 9 & bcnoxA_KF_F & $5^{\prime}-\mathrm{GAC}$ GAC CCG GGC TCG TGC ATA AGT G-3' \\
\hline 10 & bcnoxA_KF_R & 5'-CCA CTC AAC CCC GGG ATT TCC GGC TC-3' \\
\hline 11 & BcNoxF1 & $5^{\prime}$-GGG ATT TCT GGA ACG AAG ATG GGA-3' \\
\hline 12 & BcNoxF2 & 5'-GGG TAG TCA GTT GCA TAT CAA GTG-3' \\
\hline 13 & BcNoxR2 & 5'-CAT CTG AAC GAC AGC CAA TTT CC-3' \\
\hline 14 & bcNoxFl1_F & $5^{\prime}$-TGT TCT GGT ACC CCG TCA CAT-3' \\
\hline 15 & bcNoxFl1_R & 5'-CGT GTC GAC CTG GAA TCG TAA T-3' \\
\hline 16 & bcNoxFl2_F & 5'-CAT TAC GAA GAA GCT TGG CTT C- $3^{\prime}$ \\
\hline 17 & bcNoxFl2_R & 5'-GGA GAA AAT CGA ATT CGC TCA AG-3' \\
\hline 18 & bcnoxB_KF_F & 5'-GTA TGG CAT GGT CGA CGT GTG GCG AG-3' \\
\hline 19 & bcnoxB_KF_R & $5^{\prime}$-CCG GGA GTC GAC AA TGA TAT TCA TC- $3^{\prime}$ \\
\hline 20 & bcnoxA_RT_F & 5'-GAA GGA GCA GAC TCG GGG AAC TAA G-3' \\
\hline 21 & bcnoxA_RT_R & 5'-GAT GGA GCG GGC TGC TAC GTT TGG-3' \\
\hline 22 & p67_dWT & 5'-CAA AGA CAT GAT GCC TTT TCT CCC-3' \\
\hline 23 & p67_d1 & 5'-ATC GTT GTC GGG GTT AGC AGG GAG C-3' \\
\hline 24 & pLOF_OliP & 5'-GGT ACT GCC CCA CTT AGT GGC AGC TCG CG-3' \\
\hline 25 & Tub-T2 & $5^{\prime}$-GGT CCT CGG AGT GCA GAT GGG-3' \\
\hline 26 & p67_d2 & 5'-GAT CAC CCA TCG TAA TCA TGT CGC-3' \\
\hline 27 & Bcphox_FlaAFw & 5'-CAC CAT CGA AGT TGT CGA GCT CGC ATC-3' \\
\hline 28 & Bcphox_FlaARev & 5'-GTG CGA AGC TAT CTA GAA TTG TCG G-3' \\
\hline 29 & Bcphox_FlaBFw & 5'-GAT TGT CAT TCA AAG CTT CAT GTT GG-3' \\
\hline 30 & Bcphox_FlaBRev & 5'-CTT TAA TCT TGA GTC GAC CAC GCA GC-3' \\
\hline
\end{tabular}


by PCR using primer pair 18/19 (Table 1). Construction of the complementation vector was as described for bcnoxA, using SalI instead of SmaI and SacI instead of SacII.

For generation of a double mutant, $\triangle$ bcnoxB-3 was transformed with the replacement vector of bcnoxA. Three transformants with resistance against nourseothricin and hygromycin were obtained, which showed the diagnostic PCR fragments for replacement of both bcnox genes. Homokaryotic derivatives were obtained from two primary transformants showing only the diagnostic fragment and lacking the wild-type fragment (data not shown). The double mutants were named $\triangle$ bcnoxAB1 and $\triangle$ bcnoxAB-2.

A replacement vector for bcnoxR was designed and primer pairs 27/28 and 29/30 (Table 1) were used to clone the $5^{\prime}$ and $3^{\prime}$ flanking regions of bcnoxR. The flanking regions were cloned in vector pCR2.1, excised with SacI/XbaI and HindIII/SalI, respectively, and cloned into vector pOLiHP (Rolke et al. 2004). The replacement fragment was excised with $\mathrm{SacI} / \mathrm{SalI}$. Correct integration of the replacement fragment was checked by PCR with primers p67_d1/pLOF_OliP (left flank) and Tub-T2/P67_d2 (right flank), and presence of the wild-type fragment in primary transformants was checked with primers p67_d1/p67_dWT.

\section{Transformation of $\boldsymbol{B}$. cinerea.}

Protocols for protoplast formation and transformation were adapted from ten Have and associates (1998) as described by Schulze Gronover and associates (2001).

\section{Pathogenicity assays.}

Primary leaves of Phaseolus vulgaris L. genotype N90598 (originating from J. D. Kelly, Michigan State University, East Lansing U.S.A.) were inoculated with $7.5 \mu \mathrm{l}$ of conidial suspensions $\left(2 \times 10^{5}\right.$ conidia $\left.\mathrm{ml}^{-1}\right)$ of $B$. cinerea strains for the standard pathogenicity test as described by Klimpel and associates (2002). The infected plants were incubated in a plastic propagator box at $20^{\circ} \mathrm{C}$ under natural illumination. Disease symptoms were scored at 24 and 48 hpi and $3 \mathrm{dpi}$.

\section{Penetration assay.}

Onion epidermis was harvested and incubated for $1.5 \mathrm{~h}$ at $70^{\circ} \mathrm{C}$. The epidermis then was washed with sterile water and put on water agar plates. Conidia of the bcnox mutants and the wild type were harvested and washed twice with sterile water. The onion epidermis than was inoculated with $10 \mu \mathrm{l}$ of conidial suspension $\left(5 \times 10^{3}\right.$ conidia $\left.\mathrm{ml}^{-1}\right)$ of the bcnox mutants and the wild type. The infected onion epidermis was incubated at $20^{\circ} \mathrm{C}$ under natural illumination. Infection structures were analyzed 24 hpi by use of bright-field microscopy after staining of the samples with Lactophenol Blue (Merck).

\section{ROS detection assay.}

For superoxide detection, $B$. cinerea conidia were collected from 7-day CM agar plates with sterile water and filtered through sterile Nytex. A droplet of conidial suspension was placed on a microscope slide covered with CM media. Alternatively, the conidial suspension was diluted to $5 \times 10^{3}$ conidia $\mathrm{ml}^{-1}$ and droplets of $10 \mu \mathrm{l}$ were placed on onion epidermis, as described above for the penetration assays. Cultures were incubated at $20^{\circ} \mathrm{C}$ under natural illumination for $24 \mathrm{~h}$ (penetration) or $48 \mathrm{~h}$ (hyphae staining). Samples then were covered with 50 $\mathrm{mM} \mathrm{NaPO} 4$ buffer, $\mathrm{pH} 7.5$, containing $0.05 \% \mathrm{NBT}$ (wt/vol) for $4 \mathrm{~h}$ (hyphae staining) or overnight (penetration). As controls, samples were preincubated for $20 \mathrm{~min}$ in $50 \mu \mathrm{M}$ DPI (Sigma).

\section{Transmission electron microscopy.}

For $\mathrm{H}_{2} \mathrm{O}_{2}$ detection, leaf discs of $P$. vulgaris inoculated with $\Delta$ noxA-5, $\Delta$ bcnoxB-3, $\Delta$ bcnoxAB-1, and wild-type strain
B05.10 (24 and $48 \mathrm{hpi}$ ) were vacuum infiltrated in $5 \mathrm{mM}$

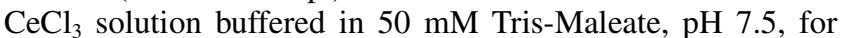
$1.5 \mathrm{~h}$ (Bestwick et al. 1997; Veenhuis et al. 1976). The solution was changed once during this incubation step. The samples were washed once with $25 \mathrm{mM}$ sodium-phosphate buffer, $\mathrm{pH}$ 6 , for $30 \mathrm{~min}$ at room temperature (at approximately $20^{\circ} \mathrm{C}$ ) and another time with $25 \mathrm{mM}$ sodium-phosphate buffer, $\mathrm{pH} 7$, for $10 \mathrm{~min}$ at room temperature. The samples then were prefixed in $3 \%$ glutaraldehyde buffered with $25 \mathrm{mM}$ phosphate buffer, $\mathrm{pH} 7$, for $2 \mathrm{~h}$ at $4^{\circ} \mathrm{C}$. Afterward, glutaraldehyde was changed and the incubation step was repeated. The samples then were washed three times with $25 \mathrm{mM}$ sodium-phosphate buffer, $\mathrm{pH}$ 7 , for $20 \mathrm{~min}$ at $4^{\circ} \mathrm{C}$. Subsequently, the samples were fixed in $2 \%$ osmium tetroxide buffered with $25 \mathrm{mM}$ sodium-phosphate buffer, $\mathrm{pH} 7$, for $2 \mathrm{~h}$ at room temperature. After two washing steps with $25 \mathrm{mM}$ sodium-phosphate buffer, $\mathrm{pH} \mathrm{7,} \mathrm{for} 20 \mathrm{~min}$ at $4{ }^{\circ} \mathrm{C}$, the samples were dehydrated with $\mathrm{EtOH}$ and propyleneoxide and then embedded in epoxy resin as described by Spurr (1969).

\section{Sexual crosses.}

Crosses were performed as described by van der VlugtBergmans and associates (1993). Cap structures of mature apothecia were collected and placed on a glass microscope slide in a droplet of sterile water. The cap was crushed with a second microscope slide in order to release asci and ascospores. The sample was suspended in a 2 to $3 \mathrm{ml}$ of sterile water and filtered over glasswool to remove large debris. The resulting flowthrough contained a suspension of ascospores which was plated on appropriate media for further analysis.

\section{Bioinformatic tools.}

Protein sequences were aligned by the neighbor-joining method using the computer program MegAlign (DNAStar Inc.) with ClustalW (default parameters). The default parameters were as follows: for multiple alignments, the gap penalty was set to 10.00 and the gap length penalty to 0.20 ; for the pairwise alignment, the gap penalty was set to 10.00 and the gap length to 0.10 . The used matrix was Gonnet 250 .

\section{ACKNOWLEDGMENTS}

This work was supported by the Deutsche Forschungsgemeinschaft (Tu50/14). We thank B. Williamson (Dundee) for critical reading of the manuscript, J. Schumacher for discussion, and M. Hahn (Universität Kaiserslautern) for providing the MAPK mutants $\Delta$ bmp1/3.

\section{LITERATURE CITED}

Aguirre, J., Rios-Momberg, M., Hweitt, D., and Hansberg, W. 2005. Reactive oxygen species and development in microbial eukaryotes. Trends Microbiol. 13:111-118.

Arnold, R. S., Shi, J., Murad, E., Whalen, A. M., Sun, C. Q., Polavarapu, R., Parthasarathy, S., Petros, J. A., and Lambeth, J. D. 2001. Hydrogen peroxide mediates the cell growth and transformation caused by the mitogenic oxidase Nox1. Proc. Natl. Acad. Sci., U.S.A. 98:5550-5555.

Babior, B. M. 1992. The respiratory burst oxidase. Adv. Enzymol. Relat. Areas Mol. Biol. 65:49-95.

Baker, C. J., and Orlandi, E. W. 1995. Active oxygen in plant pathogenesis. Annu. Rev. Phytopathol. 33:299-321.

Bedard, K., and Krause, K.-H. 2007. Nox family NADPH oxidases: Not just in mammals. Physiol. Rev. 87:245-313.

Bestwick, C. S., Brown, I. R., Bennett, M. H., and Mansfield, J. W. 1997. Localization of hydrogen peroxide accumulation during the hypersensitive reaction of lettuce cells to Pseudomonas syringae pv. phaseolicola. Plant Cell 9:209-221.

Briggs, R. T., Drath, D. B., Karnovsky, M. L., and Karnovski, M. J. 1975. Localization of NADH oxidase on the surface of human polymorphonuclear leukocytes by a new cytochemical method. J. Cell Biol. 67:566-586.

Cenis, J. L. 1992. Rapid extraction of fungal DNA for PCR amplification. Nucleic Acids Res. 20:2380. 
Cross, A. R., and Jones, O. T. G. 1991. Enzyme mechanisms of superoxide production. Biochim. Biophys. Acta 1057:281-298.

Dinauer, M. 1993. The respiratory burst oxidase and the molecular genetics of chronic granulomatous disease. Crit. Rev. Clin. Lab. Sci. 30:329-369.

Doehlemann, G., Molitor, F., and Hahn, M. 2005. Molecular and functional characterization of a fructose specific transporter from the gray mold fungus Botrytis cinerea. Fungal Gen. Biol. 42:601-610.

Doehlemann, G., Berndt, B. and Hahn, M. 2006. Different signalling pathways involving a $\mathrm{G} \alpha$ protein, cAMP and a MAP kinase control germination in Botrytis cinerea conidia. Mol. Microbiol. 59:821-835.

Egan, M. J., Wang, Z.-Y., Jones, M. A., Smirnoff, N., and Talbot, J. T. 2007. Generation of reactive oxygen species by fungal NADPH oxidases is required for rice blast disease. Proc. Natl. Acad. Sci. U.S.A. 104:1177211777.

Finkel, T. 2003 Oxidant signals and oxidative stress. Curr. Opin. Cell Biol. $15: 247-254$

Foremanm, J., Demidchik, V., Bothwell, J. H. F., Mylona, P., Miedema H., Torres, M. A., Linstead, P., Costa, S., Brownlee, C., Jones, J. D. G., Davies, J. M., and Dolan, L. 2003. Reactive oxygen species produced by NADPH oxidase regulate plant cell growth. Nature 422:442446

Geiszt, M., Kopp, J. B., Varnai, P., and Leto, T. L. 2000. Identification of renox, an NAD $(\mathrm{P}) \mathrm{H}$ oxidase in kidney. Proc. Natl. Acad. Sci. U.S.A. 97:8010-8014.

Giesbert, S., Schürg, T., Scheele, S., and Tudzynski, P. The NADPH oxidase Cpnox1 is required for full pathogenicity of the ergot fungus Claviceps purpurea. Mol. Plant Pathol. In press.

Govrin, E. M., and Levine, A. 2000. The hypersensitive response facilitates plant infection by the necrotrophic pathogen Botrytis cinerea. Curr. Biol. 10:751-757.

Herve, C., Tonon, T., Cooen, J., Borre, E., and Boyen, C. 2006. NADPH oxidases in Eukaryotes: Red algae provide new hints! Curr. Genet. 49:190-204.

Klimpel, A., Schulze Gronover, C., Williamson, B., Stewart, J. A., and Tudzynski, B. 2002. The adenylate cyclase (BAC) in Botrytis cinerea is required for full pathogenicity. Mol. Plant Pathol. 3:439-450.

Kwak, J. M., Mori, I. C., Pei, Z.-M., Leonhardt, N., Torres, M. A., Dangl, J. L., Bloom, R. E., Bodde, S., Jones, J. D. G., and Schroeder, J. I. 2003. NADPH oxidase AtrbohD and AtrbohF genes function in ROSdependent ABA signaling in Arabidopsis. EMBO (Eur. Mol. Biol. Organ.) J. 22:2623-2633.

Lamb, C., and Dixon, R. A. 1997. The oxidative burst in plant disease resistance. Annu. Rev. Plant Physiol. Plant Mol. Biol. 48:251-275.

Lambeth, J. D. 2004. NOX enzymes and the biology of reactive oxygen. Nat. Rev. Immunol. 4:181-189.

Lara-Ortiz, T., Riveros-Rosas, H., and Aguirre, J. 2003. Reactive oxygen species generated by microbial NADPH oxidase NoxA regulated sexual development in Aspergillus nidulans. Mol. Microbiol. 50:12411255 .

Lyon, G. D., Goodman, B. A., and Williamson, B. 2004. Botrytis cinerea perturbs redox processes as an attack strategy in plants. Pages 119-141 in: Botrytis: Biology, Pathology and Control. Y. Elad, B. Williamson, P. Tudzynski, and N. Delen, eds. Kluwer Academic Publishers, Dordrecht, The Netherlands.

Malagnac, F., Laluque, H., Lepere, G., and Silar, P. 2004. Two NADPH oxidase isoforms are required for sexual reproduction and ascospore germination in the filamentous fungus Podospora anserina. Fungal Gen. Biol. 41:982-997.

Parkos, C. A., Allen, R. A., Cochrane, C. G., and Jesiatis, A. J. 1988. The quaternary structure of the plasma membrane $b$-type cytochrome of human granulocytes. Biochim. Biophys. Acta 932:71-83.

Pontecorvo, G. V., Roper, J. A., Hummonns, L. M., MacDonald, K. D., and Buften, A. W. J. 1953. The genetics of Aspergillus nidulans. Adv. Genet. 141:141-238.

Quidde, T., Büttner, P., and Tudzynski, P. 1999. Evidence for three different specific saponin-detoxyfying activities in Botrytis cinerea and cloning and functional analysis of a gene coding for a putative avenacinase. Eur. J. Plant Pathol. 105:273-283.

Rolke, Y., Liu, S., Quidde, T., Williamson, B., Schouten, A., Weltring, K.M., Siewers, V., Tenberge, K. B., Tudzynski, B., and Tudzynski, P. 2004. Functional analysis of $\mathrm{H}_{2} \mathrm{O}_{2}$-generating systems in Botrytis cinerea: The major $\mathrm{Cu}-\mathrm{Zn}$-superoxide dismutase (BCSOD1) contributes to virulence on French bean, whereas a glucose oxidase (BCGOD1) is dispensable. Mol. Plant Pathol. 5:17-27.
Rui, O., and Hahn, M. 2007. The Slt2-type MAP kinase Bmp3 of Botrytis cinerea is required for normal saprotrophic growth, conidiation, plant surface sensing and host tissue colonization. Mol. Plant Pathol. 8:173-184.

Sambrook, J., Fritsch, E. F., and Maniatis, T. 1989. Molecular Cloning: A Laboratory Manual, 2nd ed. Cold Spring Harbor Laboratory Press, Cold Spring Harbor, NY, U.S.A.

Schulze Gronover, C., Kasulke, D., Tudzynski, P., and Tudzynski, B. 2001 The role of $\mathrm{G}$ protein alpha subunits in the infection process of the grey mould fungus Botrytis cinerea. Mol. Plant-Microbe Interact. 14:12931302 .

Segal, A. W. 2005. How neutrophils kill microbes. Annu. Rev. Immunol. 23:197-223.

Segmüller, N., Ellendorf, U., Tudzynski, B., and Tudzynski, P. 2007. $\mathrm{BcSAK} 1$, a stress-activated mitogen-activated protein kinase is involved in vegetative differentiation and pathogenicity in Botrytis cinerea. Eukaryot. Cell 6:211-221.

Shinogi, T., Suzuki T., Kurihara, T., Narusake, Y., and Park, P. 2003. Microscopic detection of reactive oxygen species generation in the compatible and incompatible interactions of Alternaria alternata Japanese pear pathotype and host plants. J. Gen. Plant Pathol. 69:7-16.

Siewers, V., Viaud, M., Jimez-Teja, D., Collado, I. G., Schulze Gronover, C., Pradier, J.-M., Tudzynski, B., and Tudzynski, P. 2005. Functional analysis of the cytochrome P450 monooxygenase gene bcbotl of Botrytis cinerea indicates that botrydial is a strain-specific virulence factor. Mol. Plant-Microbe Interact. 18:602-612.

Spurr, A. R. 1969. A low viscosity epoxy resin embedding medium for electron microscopy. J. Ultrastruct. Res. 26:31-43.

Suh, Y. A., Arnold, R. S., Lassegue, B., Shi, J., Xu, X., Sorescu, D., Chiung, A. B., Griendling, K. K., and Lambeth, J. D. 1999. Cell transformation by the superoxide-generating oxidase Mox1. Nature 401:79-82.

Takemoto, D., Tanaka, A., and Scott, B. 2006. A p67Phox-like regulator is recruited to control hyphal branching in a fungal-grass mutualistic symbiosis. Plant Cell 18:2807-2821.

Takemoto, P., Tanaka, and A., Scott, B. 2007. NADPH oxidases in fungi: Diverse roles of reactive oxygen species in fungal cellular differentiation. Fungal Genet. Biol. 44:1065-1076.

Tanaka, A., Christensen, M. J., Takemoto, D., Park, P., and Scott, B. 2006. Reactive oxygen species play a role in regulating a fungus-perennial ryegrass mutualistic interaction. Plant Cell 18:1052-1066.

Tenberge, K. B., Beckedorf, M., Hoppe, B., Schouten, A., Solf M., and von den Driesch, M. 2002. In situ localization of AOS in host-pathogen interactions. Microsc. Microanal. 8 (Suppl. 2):250-251.

ten Have, A., Mulder, W., Visser, J., and van Kan, J. A. L. 1998. The endopolygalacturonase gene Bcpg1 is required for full virulence of Botrytis cinerea. Mol. Plant-Microbe Interact. 11:1009-1016.

Torres, M. A., Jones, J. D. G., and Dangl, J. L. 2006. Reactive oxygen species signaling in response to pathogens. Plant Physiol. 141:373-378.

van der Vlugt-Bergmans, C. J. B., Brandwagt, B. F., Van't Klooster, J. W., Wagemakers, C. A. M., and Van Kan, J. A. L. 1993. Genetic variation and segregation of DNA polymorphisms in Botrytis cinerea. Mycol. Res. 97:1193-1200.

Veenhuis, M., van Dijken, J. P., and Harder, W. 1976. Cytochemical studies on localization of methanol oxidase and other oxidases in peroxisomes of methanol-grown Hansenula polymorpha. Arch. Microbiol. 111:123-135.

von Tiedemann, A. 1997. Evidence for a primary role of active oxygen species in induction of host cell death during infection of bean leaves with Botrytis cinerea. Physiol. Mol. Plant Pathol. 50:151-166.

Wientjes, F. B., and Segal, A. W. 1995. NADPH oxidase and the respiratory burst. Semin. Cell Biol. 6:357-365.

Williamson, B, Tudzynski, B., Tudzynski, P., and vanKan, J. A. L. 2007. Botrytis cinerea: The cause of grey mould disease. Mol. Plant Pathol. 8:561-580.

Wojtaszek, P. 1997. Oxidative burst: An early plant response to pathogen infection. Biochem. J. 322 (Pt. 3):681-692.

Zheng, L., Campbell, M., Murphy, J., Lam, S., and Xu, J.-R. 2000. The BMP1 gene is essential for pathogenicity in the gray mold fungus $\mathrm{Bo}$ trytis cinerea. Mol. Plant-Microbe Interact. 13:724-732.

\section{AUTHOR-RECOMMENDED INTERNET RESOURCE}

Broad Institute's Botrytis cinerea database: www.broad.mit.edu/annotation/genome/botrytis_cinerea/Home.html 